\title{
Derivation of generalized Einstein's equations of gravitation based on a mechanical model of vacuum and a sink flow model of particles
}

\author{
Xiao-Song Wang \\ Institute of Mechanical and Power Engineering, Henan Polytechnic University, Jiaozuo, Henan Province, 454000, China \\
}

(Dated: Augest 28, 2018)

J. C. Maxwell, B. Riemann and H. Poincaré have proposed the idea that all microscopic particles are sink flows in a fluidic aether. Following this research program, a previous theory of gravitation based on a mechanical model of vacuum and a sink flow model of particles is generalized by methods of special relativistic continuum mechanics. In inertial coordinate systems, we construct a tensorial potential which satisfies the wave equation. Inspired by the equation of motion of a test particle, a definition of a metric tensor of a Riemannian spacetime is introduced. Applying Fock's theorem, a generalized Einstein's equation is derived based on some assumptions. This equation reduces to Einstein's equation in case of weak field in harmonic coordinate systems. In some special non-inertial coordinate systems, a second generalized Einstein's equation is derived based on some assumptions. If the field is weak and the coordinate system is quasi-inertial and harmonic, the second generalized Einstein's equation reduces to Einstein's equation. Thus, this theory also explains all the experiments that support the theory of general relativity. There exists some fundamental differences between this theory and Einstein's theory of general relativity.

Keywords: Einstein's equation; gravitation; general relativity; sink; gravitational aether. 


\section{INTRODUCTION}

The Einstein's equation of gravitational fields in the theory of general relativity can be written as [1-4]

$$
R_{\mu \nu}-\frac{1}{2} g_{\mu \nu} R=-\kappa T_{\mu \nu}^{m}
$$

where $g_{\mu \nu}$ is the metric tensor of a Riemannian spacetime, $R_{\mu \nu}$ is the Ricci tensor, $R \equiv g^{\mu \nu} R_{\mu \nu}$ is the scalar curvature, $g^{\mu \nu}$ is the contravariant metric tensor, $\kappa=8 \pi \gamma_{N} / c^{4}, \gamma_{N}$ is Newton's gravitational constant, $c$ is the speed of light in vacuum, $T_{\mu \nu}^{m}$ is the energy-momentum tensor of a matter system.

The Einstein's equation (1) is a fundamental assumption in the theory of general relativity [1-4]. It is remarkable that Einstein's theory of general relativity, born 102 years ago, has held up under every experimental test [5-9]. R. P. Feynman once said:"What I cannot create, I do not understand." ([10], page xxxii). New theories which can derive Einstein's equation Eq.(1) and explain all known experiments of gravitational phenomena may be interesting. The reasons may be summarized as follows.

(1). Many attempts to reconcile the theory of general relativity and quantum mechanics by using the techniques in quantum electrodynamics meet some mathematical difficulties ([11],p101). J. Maddox speculates that the failure of the familiar quantization procedures to cope with Einstein' equation may stem from two possible reasons. One possibility is that Einstein' equation are incomplete. The other is that some underlying assumptions about the character of the space or time may faulty ([11],p101).

(2). The value of the cosmological constant is a puzzle [12]. In 1917, Einstein thought that Eq.(1) should be revised as $([3], \mathrm{p} 410)$

$$
R_{\mu \nu}-\frac{1}{2} g_{\mu \nu} R+\Lambda g_{\mu \nu}=-\kappa T_{\mu \nu}^{m}
$$

where $\Lambda$ is the cosmological constant. However, it seems that the cosmological constant $\Lambda$ is unnecessary when Hubble discovered the expansion of the universe. Thus, Einstein abandoned Eq.(2) and returned to Eq.(1) ([3], p410). The value of the cosmological constant $\Lambda$ is also related to the energy-momentum tensor of vacuum ([3], p411). Theoretical interpretation of the small value of $\Lambda$ is still open [12].

(3). The problem of the existence of black hole is still controversy [13]. Einstein believed that black hole can not exist in the real world [14].

(4). P. A. M. Dirac's dimensionless large number ([15], p73)

$$
\frac{e^{2}}{G m_{e} m_{p}} \sim 2 \times 10^{39}
$$

where $e$ is the charge of an electron, $m_{e}$ is the mass of an electron, $m_{p}$ is the mass of a proton, is still a puzzle. Dirac speculates that this large number is related to the age of the Universe ([15], p74).

(5). The existences and characters of dark matter dark energy are still controversy, refers to, for instance, [16-20].

(6). The existence and characters of gravitational aether are still not clear. Sir I. Newton pointed out that his inverse-square law of gravitation did not touch on the mechanism of gravitation ([21],p28;[22],p91). Newton warned ([23], p204):" That Gravity should be innate, inherent and essential to Matter, so that one Body may act upon another at a Distance thro' a Vacuum, without the Mediation of any thing else, by and through which their Action and Force may be conveyed from one to another, is to me so great an Absurdity, that I believe no Man who has in philosophical Matters a competent Faculty of thinking, can ever fall into it. " He conjectured that gravitation may be explained based on the action of an aether pervading the space ([21],p28;[22],p92). In the years 1905-1916, Einstein abandoned the concepts of electromagnetic aether and gravitational aether in his theory relativity ([24], p27-61). However, H. A. Lorentz believed that general relativity could be reconciled with the concept of an ether at rest and wrote a letter to A. Einstein ([24], p65). Einstein changed his view later and introduced his new concept of ether ([24], p63-113). In 1920, Einstein said ([24], p98):" According to the general theory of relativity, space is endowed with physical qualities; in this sense, therefore, there exists an ether. According to the general theory of relativity, space without ether is unthinkable;". In 1954, Einstein said ([24], p149):" There is no such thing as an empty space, i.e., a space without field. Space-time does not claim existence on its own, but only as a structural quality of the field." Unfortunately, Einstein did not tell us how to derive his equation (1) theoretically based on his new concept of the gravitational aether. Nor did he tell us how to detect his gravitational aether by experiments.

(7). Whether Newton's gravitational constant $\gamma_{N}$ depends on time and space is still not clear. It is known that $\gamma_{N}$ is a constant in Newton's and Einstein's theory of gravitation. P. A. M. Dirac speculates that $\gamma_{N}$ may depend on time based on his large number hypothesis [25]. R. P. Feynman thought that if $\gamma_{N}$ decreases on time, then the 
earth's temperature a billion years ago was about $48^{\circ} \mathrm{C}$ higher than the present temperature ([10], p9). D. R. Long reports that $\gamma_{N}$ depends on the distance away from the surface of the earth [26].

(8). We are facing a puzzle that whether the speed of light in vacuum is a constant, refers to, for instance, [27-29].

Furthermore, there exists some other problems related to the theories of gravity, for instance, the definition of inertial system, origin of inertial force, the velocity of the propagation of gravity [30], the velocity of individual photons [28, 29], unified field theory, etc.

There is a long history of researches of derivations or interpretations of Einstein's theory of general relativity. C. Misner et al. introduce six derivations of the Einstein's equation Eq.(1) in their great book ([3], p417). S. Weinberg proposed two derivations ([2], p151).

However, these theories still face the aforementioned difficulties. Thus, it seems that new ideas about the gravitational phenomena are needed. The gravitational interaction seems to differ in character from other interactions. In 1949, Einstein wrote in a letter to Solovine [31]:" I am not convinced of the certainty of a simple concept, and I am uncertain as to whether I was even on the right track." Thus, following Einstein, it is better for us to keep an open and critical mind to review all possible theories about gravity.

There exists some approaches ([10], page vii;[3], p424), which regards Einstein's general relativity as a special relativistic field theory in an unobservable flat spacetime, to derive the Einstein's equation (1). Now we briefly review the history of the theories of gravitation in flat spacetime. According to J. Preskill and K. S. Thorne ([10], page viii), the field equation for a free massless spin-2 field was proposed by Fierz and Pauli in the year 1939. Since then, the idea of treating Einstein's theory of gravity as a massless spin 2 field theory in flat spacetime appeared in the literature. In 1954, S. N. Gupta finds that some features of Einstein's theory of gravity are a necessary consequence of the fact that Einstein's field corresponds to particles of massless spin 2 [32]. In 1955, R. H. Kraichnan proposes a special relativistic derivation of generally covariant gravitation theory [33]. In 1961, W. Thirring proposes an alternative tensor field theory of gravitation in flat spacetime based on a specific Lagrangian [34]. R. P. Feynman constructs the quadratic action of a massless spin 2 field that is linearly coupled to a conserved energy-momentum tensor ([10], p43). Then, he derive the Einstein's equation (1) by requiring that the field equation be compatible with the matter's equation of motion ([10], Chapter 6). In 1965, starting from the Lorentz invariance of the S matrix calculated by Feynman-Dyson perturbation theory, S. Weinberg shows that Maxwell's theory of electromagnetism and Einstein's theory of gravitation are essentially the unique Lorentz-invariant theories of massless particles with spin 1 and spin 2 [35]. In 1970, S. Deser [36] derives the Einstein's equation (1) based on the self-coupling of the gravitational fields with just one added cubic term without special gauge condition, rather than as an infinite series [32]. In 1999, S. V. Babak and L. P. Grishchuk treat gravity as a nonlinear tensor field in flat spacetime and derive the Einstein's equation (1) based on a specific Lagrangian [37]. It is impossible for us to list all the works related to theories of gravitation in flat spacetime here.

However, these theories $[3,10,32-37]$ can not provide a physical definition of the tensorial potential of gravitational fields. Thus, similar to the theory of general relativity, these theories may be regarded as phenomenological theories of gravitation.

The purpose of this manuscript is to propose a derivation of the Einstein's equation (1) based on a mechanical model of vacuum and a sink flow model of particles [38].

\section{INTRODUCTION OF A PREVIOUS THEORY OF GRAVITATION BASED ON A SINK FLOW MODEL OF PARTICLES BY METHODS OF CLASSICAL FLUID MECHANICS}

The idea that all microscopic particles are sink flows in a fluidic substratum is not new. For instance, in order to compare fluid motions with electric fields, J. C. Maxwell introduced an analogy between source or sink flows and electric charges ([21], p243). B. Riemann speculates that:"I make the hypothesis that space is filled with a substance which continually flows into ponderable atoms, and vanishes there from the world of phenomena, the corporeal world" ([39], p507). H. Poincaré also suggests that matters may be holes in fluidic aether ([40], p171). A. Einstein and L. Infeld said ([41], p256-257):" Matter is where the concentration of energy is great, field where the concentration of energy is small. ... What impresses our senses as matter is really a great concentration of energy into a comparatively small space. We could regard matter as the regions in space where the field is extremely strong."

Following these researchers, we suppose that all the microscopic particles were made up of a kind of elementary sinks of $\Omega(0)$ substratum [38]. Thus, Newton's law of gravitation is derived by methods of hydrodynamics based on the fluid model of vacuum and the sink flow model of particles [38].

We briefly introduce this theory of gravitation [38]. Suppose that there exists a fluidic medium filling the interplanetary vacuum. We may call this medium as the $\Omega(0)$ substratum, or gravitational aether, or tao [38]. Suppose that the following conditions are valid: (1) the $\Omega(0)$ substratum is an ideal fluid; (2) the ideal fluid is irrotational and 


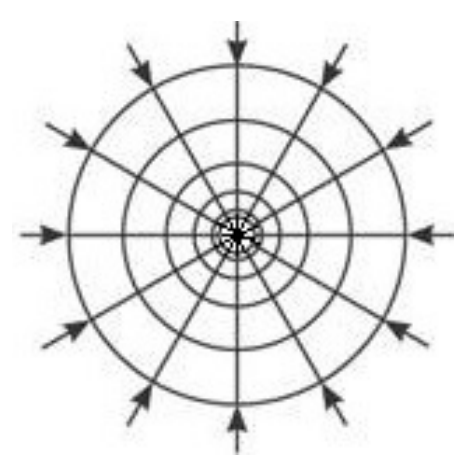

FIG. 1. an illustration the velocity field of a sink flow.

barotropic; (3) the density of the $\Omega(0)$ substratum is homogeneous; (4) there are no external body forces exerted on the fluid; (5) the fluid is unbounded and the velocity of the fluid at the infinity is approaching to zero.

An illustration of the velocity field of a sink flow can be found in Figure 1. If a point source is moving with a velocity $\mathbf{v}_{s}$, then there is a force [38]

$$
\mathbf{F}_{Q}=-\rho_{0} Q\left(\mathbf{u}-\mathbf{v}_{s}\right)
$$

is exerted on the source by the fluid, where $\rho_{0}$ is the density of the fluid, $Q$ is the strength of the source, $\mathbf{u}$ is the velocity of the fluid at the location of the source induced by all means other than the source itself.

We suppose that all the elementary sinks were created simultaneously [38]. For convenience, we may call these elementary sinks as monads. The initial masses and the strengths of the monads are the same. Suppose that (1) $\mathbf{v}_{i} \ll \mathbf{u}_{i}, i=1,2$, where $\mathbf{v}_{i}$ is the velocity of the particle with mass $m_{i}, \mathbf{u}_{i}$ is the velocity of the $\Omega(0)$ substratum at the location of the particle with mass $m_{i}$ induced by the other particle; (2) there are no other forces exerted on the particles, then the force $\mathbf{F}_{21}(t)$ exerted on the particle with mass $m_{2}(t)$ by the velocity field of $\Omega(0)$ substratum induced by the particle with mass $m_{1}(t)$ is [38]

$$
\mathbf{F}_{21}(t)=-\gamma_{N}(t) \frac{m_{1}(t) m_{2}(t)}{r^{2}} \hat{\mathbf{r}}_{21},
$$

where $\hat{\mathbf{r}}_{21}$ denotes the unit vector directed outward along the line from the particle with mass $m_{1}(t)$ to the particle with mass $m_{2}(t), r$ is the distance between the two particles, $m_{0}(t)$ is the mass of monad at time $t,-q_{0}\left(q_{0}>0\right)$ is the strength of a monad, and

$$
\gamma_{N}(t)=\frac{\rho_{0} q_{0}^{2}}{4 \pi m_{0}^{2}(t)}
$$

For continuously distributed matter, we have

$$
\frac{\partial \rho_{0}}{\partial t}+\nabla \cdot\left(\rho_{0} \mathbf{u}\right)=\rho_{0} \rho_{s},
$$

where $\mathbf{u}$ is the velocity of the $\Omega(0)$ substratum, $\nabla=\mathbf{i} \partial / \partial x+\mathbf{j} \partial / \partial y+\mathbf{k} \partial / \partial z$ is the Hamilton operator, $\mathbf{i}, \mathbf{j}, \mathbf{k}$ are basis vectors, $\rho_{s}$ is the density of continuously distributed sinks, i.e.,

$$
\rho_{s}=\lim _{\triangle V \rightarrow 0} \frac{\triangle Q}{\triangle V},
$$

where $\triangle Q$ is the source strength of the continuously distributed matter in the volume $\triangle V$ of the $\Omega(0)$ substratum.

Since the $\Omega(0)$ substratum is homogeneous, i.e., $\partial \rho_{0} / \partial t=\partial \rho_{0} / \partial x=\partial \rho_{0} / \partial y=\partial \rho_{0} / \partial z=0$, and irrotational, i.e., $\nabla \times \mathbf{u}=0$, Eq.(7) can be written as $[42-44]$

$$
\nabla^{2} \varphi=\rho_{s},
$$

where $\varphi$ is a velocity potential such that $\mathbf{u}=\nabla \varphi, \nabla^{2}=\partial^{2} / \partial x^{2}+\partial^{2} / \partial y^{2}+\partial^{2} / \partial z^{2}$ is the Laplace operator.

We introduce the following definitions

$$
\Phi=\frac{\rho_{0} q_{0}}{m_{0}} \varphi, \rho_{m}=-\frac{m_{0} \rho_{s}}{q_{0}},
$$


where $\rho_{m}$ denotes the mass density of continuously distributed particles.

Using Eq.(10) and Eq.(6), Eq.(9) can be written as

$$
\nabla^{2} \Phi=-4 \pi \gamma_{N} \rho_{m}
$$

Although the new formula of gravitation Eq.(5) is similar to Newton's inverse-square-law of gravitation, there exists at least the following 5 differences between this theory [38] and Newton's theory [45].

(1). The gravitational masses are constants in Newton's law. In this theory [38], the gravitational masses are functions of time $t$.

(2). The gravitational constant $\gamma_{N}$ is a constant in Newton's theory. In this theory [38], the parameter $\gamma_{N}$ in Eq. $(6)$ depends on time $t$.

(3). In this theory, the gravitational $\gamma_{N}$ depends on the density $\rho_{0}$ of the $\Omega(0)$ substratum. If $\rho_{0}$ varies from place to place, i.e., $\rho=\rho(t, x, y, z)$, then the space dependence of the gravitational constant $\gamma_{N}$ can be seen from Eq. $(6)$.

(4). In Newton's theory of gravitation, the gravity is action-at-a-distance [46]. In this theory [38], the gravity is transmitted by the $\Omega(0)$ substratum.

(5). Newton's law of gravitation is an assumption. In this theory [38], Eq.(5) is derived by methods of classical fluid mechanics based on some assumptions.

\section{A MECHANICAL MODEL OF VACUUM}

A. Einstein strongly believe that there is no empty space ([24], p149). He thought that physical space and the aether are the same things and we could not be able to do without the aether in theoretical physics ([24], p188).

In 2008, we suppose that vacuum is filled with a kind of continuously distributed material which may be called $\Omega(1)$ substratum or electromagnetic aether [47]. We speculate that the $\Omega(1)$ substratum might behave like a fluid with respect to translational motion of large bodies through it, but would still posses elasticity to produce small transverse vibrations. Thus, Maxwell's equation in vacuum are derived by methods of continuum mechanics based on this mechanical model of vacuum and a source and sink flow model of electric charges [47].

We speculate that the electromagnetic aether may also generate gravity. Thus, we introduce the following assumption.

Assumption 1 The particles that constitute the $\Omega(1)$ substratum, or the electromagnetic aether, are sinks in the $\Omega(0)$ substratum, or the gravitational aether.

Then, according to the previous theory of gravitation [38], these $\Omega(1)$ particles gravitate with each other and also attract with matters. Thus, vacuum is composed of at least two kinds of interacting substratums, i.e., the gravitational aether $\Omega(0)$ and the electromagnetic aether $\Omega(1)$.

From Eq.(4), we see that there exists a universal damping force [38]

$$
\mathbf{F}_{d}=-\frac{\rho_{0} q_{0}}{m_{0}} m \mathbf{v}_{p}
$$

where $\mathbf{v}_{p}$ is the velocity of the particle, exerted on each particle by the $\Omega(0)$ substratum.

In 2014, we suppose that there is also a stochastic force exerting on each microscopic particle by the gravitational aether $\Omega(0)$ [48]. Then we derive a generalized Schrödinger equation for microscopic particles based on the fluidic continuum model of vacuum and the sink model of particles. Only in special cases this nonlinear generalized Schrödinger equation reduces to the usual linear Schrödinger equation. As a byproduct, the Planck constant $h$ is calculated theoretically. Thus, quantum phenomena may also be interpreted based on the gravitational aether $\Omega(0)$.

For convenience, we may call these theories $[38,47,48]$ as the theory of vacuum mechanics. If the Einstein's equation (1) of gravitational fields can be derived based on the theory of vacuum mechanics [38, 47, 48], then, electromagnetic phenomena, gravitational phenomena and quantum phenomena may be understood in this unified theory.

\section{CONSTRUCTION OF A LAGRANGIAN FOR FREE FIELDS OF THE $\Omega(0)$ SUBSTRATUM BASED ON A TENSORIAL POTENTIAL IN THE GALILEAN COORDINATES}

Inspired by the special relativistic field theories of gravitation [3, 10, 32-37], we explore the possibility of establishing a similar theory based on the theory of vacuum mechanics [38, 47, 48]. Thus, first of all, we need to construct a Lagrangian for free fields of the $\Omega(0)$ substratum based on a tensorial potential in the Galilean coordinates. In this 
section, we will regard the $\Omega(0)$ substratum in the previous theory of gravitation [38] as a special relativistic fluid. Then, we will study the $\Omega(0)$ substratum by methods of special relativistic continuum mechanics [1].

In this article, we adopt the mathematical framework of the theory of special relativity $[1,2,49]$. However, the physical interpretation of the mathematics of the theory of special relativity may be different from Einstein's theory.

We introduce the following Galilean coordinate system

$$
x^{0} \equiv t, x^{1} \equiv x, x^{2} \equiv y, x^{3} \equiv z,
$$

where $\{o, x, y, z\}$ is a Cartesian coordinate system for a three-dimensional Euclidean space that attached to the $\Omega(1)$ substratum, $\{o, t\}$ is a one-dimensional time coordinate.

According to Fock's theorem of characteristics ([50], p432), the characteristics of the generalized wave equation, or d'Alembert equation

$$
\frac{1}{\sqrt{-\gamma}} \frac{\partial}{\partial x^{\alpha}}\left(\sqrt{-\gamma} \gamma^{\alpha \beta} \frac{\partial \psi}{\partial x^{\beta}}\right)=0
$$

is

$$
\gamma_{\mu \nu} \frac{\partial \omega}{\partial x^{\mu}} \frac{\partial \omega}{\partial x^{\nu}}=0
$$

where $\psi$ is a field variable, $\gamma_{\alpha \beta}$ is a metric tensor, $\gamma=$ Det $\gamma_{\alpha \beta}, \omega\left(x_{0}, x_{1}, x_{2}, x_{3}\right)$ is the characteristics.

It is known that Maxwell's equation are valid in the frame of reference that attached to the $\Omega(1)$ substratum [47]. We denote this reference frame as $S_{\Omega(1)}$. Based on the Maxwell's equation, the law of propagation of an electromagnetic wave front in this reference frame $S_{\Omega(1)}$ can be derived and can be written as ([50],p13)

$$
\frac{1}{c^{2}}\left(\frac{\partial \omega}{\partial t}\right)^{2}-\left(\frac{\partial \omega}{\partial x}\right)^{2}-\left(\frac{\partial \omega}{\partial y}\right)^{2}-\left(\frac{\partial \omega}{\partial z}\right)^{2}=0
$$

where $\omega\left(x^{0}, x^{1}, x^{2}, x^{3}\right)$ is an electromagnetic wave front, $c$ is the velocity of light in the reference frame $S_{\Omega(1)}$.

An electromagnetic wave front is a characteristics. Comparing Eq.(16) and Eq.(15), we obtain the following metric tensor $\eta_{\alpha \beta}$ of a Minkowski spacetime for vacuum ([49], p57)

$$
\eta_{\alpha \beta}=\operatorname{diag}\left[c^{2},-1,-1,-1\right] .
$$

For convenience, we introduce the following Galilean coordinate system

$$
x^{0} \equiv c t, x^{1} \equiv x, x^{2} \equiv y, x^{3} \equiv z .
$$

We will use Greek indices $\alpha, \beta, \mu, \nu$, etc., denote the range $\{0,1,2,3\}$ and use Latin indices $i, j$, $k$, etc., denote the range $\{1,2,3\}$. We introduce the following definition of spacetime interval

$$
d s^{2}=\sum_{\mu=0}^{3} \sum_{\nu=0}^{3} \eta_{\mu \nu} d x^{\mu} d x^{\nu},
$$

where $\eta_{\mu \nu}$ is a matrix defined by

$$
\eta_{\mu \nu}=\left(\begin{array}{rrrr}
1 & 0 & 0 & 0 \\
0 & -1 & 0 & 0 \\
0 & 0 & -1 & 0 \\
0 & 0 & 0 & -1
\end{array}\right)
$$

$\eta_{\mu \nu}$ is the metric tensor of the Minkowski spacetime. We will use Einstein's summation convention, that is, any repeated Greek superscript or subscript appearing in a term of an equation is to be summed from 0 to 3 . Thus, Eq.(19) can be written as

$$
d s^{2}=\eta_{\mu \nu} d x^{\mu} d x^{\nu}
$$

The inverse matrix of $\eta_{\mu \nu}$ is defined by

$$
\eta^{\nu \lambda} \eta_{\lambda \mu}=\delta_{\mu}^{\nu}
$$


where $\delta_{\mu}^{\nu}$ is the Kronecker delta, $\mu, \nu, \lambda=0,1,2,3$.

Suppose that the $\Omega(0)$ substratum is an incompressible viscous fluid. Then, there is no elastic deformations in the fluid and the internal stress states depend on the instantaneous velocity field. Thus, we can choose the reference frame $S_{\Omega(1)}$ as the co-moving coordinate system. The internal energy $U$ is the sum of the internal elastic energy $U_{e}$ and the dissipative energy $U_{d}$, i.e., $U=U_{e}+U_{d}$. Since there is no elastic deformations in the fluid, we have $U_{e}=0$. We introduce the following definition of deviatoric tensor of strain rate $\dot{\gamma}_{j}^{i}([51], \mathrm{p} 331)$

$$
\dot{\gamma}_{j}^{i}=\dot{S}_{j}^{i}-\dot{S}_{k}^{k} \delta_{j}^{i}
$$

where $\dot{S}_{j}^{i}$ is the tensor of strain rate, $\dot{S}_{k}^{k}$ is the rate of volume change, $\delta_{j}^{i}$ is the Kronecker delta, $\mathrm{i}, \mathrm{j}, \mathrm{k}=1,2,3$.

Suppose that the rate of dissipative energy $\dot{U}_{d}$ is the Rayleigh type, then, we have ([51],p332)

$$
\dot{U}_{d}=\mu_{0} \dot{\gamma}_{j}^{i} \dot{\gamma}_{i}^{j}
$$

where $\mu_{0}$ is the coefficient of viscosity.

Since the $\Omega(0)$ substratum is incompressible, we have $\dot{S}_{k}^{k}=0$. Thus, from Eq.(24) and Eq.(23), we have

$$
\dot{U}_{d}=\mu_{0} \dot{S}_{j}^{i} \dot{S}_{i}^{j}
$$

In the nonrelativistic limit, i.e., $u / c \ll 1$, where $u=|\mathbf{u}|$, the Lagrangian $L_{0}$ for free fields of the $\Omega(0)$ substratum can be written as ([51],p332)

$$
L_{0}=\frac{1}{2} \rho_{0} u^{2}+\int_{t_{0}}^{t} \dot{U}_{d}\left(\dot{S}_{j}^{i}\right) d t
$$

where $u=|\mathbf{u}|, t_{0}$ is an initial time.

Suppose that the $\Omega(0)$ substratum is a newtonian fluid and the stress tensor $\sigma_{j}^{i}$ is symmetry, then we have ([51],p333)

$$
\sigma_{j}^{i}=-p \delta_{j}^{i}+2 \mu_{0} \dot{S}_{j}^{i}
$$

where $p$ is the pressure of the $\Omega(0)$ substratum, $i, j, k=1,2,3$.

Using Eq.(27) and Eq.(25), Eq.(26) can be written as

$$
L_{0}=\frac{1}{2} \rho_{0} u^{2}+\int_{t_{0}}^{t}\left(\sigma_{j}^{i}+p \delta_{j}^{i}\right) \frac{\dot{S}_{i}^{j}}{2} d t,
$$

For a macroscopic observer, the relaxation time $t_{r}$ of the $\Omega(0)$ substratum is so small that the tensor of strain rate $\dot{S}_{j}^{i}$ may be regarded as a slow varying function of time, i.e., $\left|\partial \dot{S}_{j}^{i} / \partial t\right| \ll 1$. Thus, in a small time interval $\left[t_{0}, t\right]$, we have $\dot{S}_{j}^{i} \geq 0$, or, $\dot{S}_{j}^{i} \leq 0$. Then, it is possible to choose a value $\bar{\sigma}_{j}^{i}+\bar{p} \delta_{j}^{i}$ of $\sigma_{j}^{i}+p \delta_{j}^{i}$ in the time interval $\left[t_{0}, t\right]$ such that Eq.(28) can be written as

$$
L_{0}=\frac{1}{2} \rho_{0} u^{2}+\left(\bar{\sigma}_{j}^{i}+\bar{p} \delta_{j}^{i}\right) \int_{t_{0}}^{t} \frac{\dot{S}_{i}^{j}}{2} d t .
$$

We introduce the following definition

$$
\psi_{i j} \stackrel{\text { def }}{=} \int_{t_{0}}^{t} \frac{\dot{S}_{i j}}{2 f_{0}} d t,
$$

where $f_{0}$ is a parameter to be determined.

Using Eq.(30), Eq.(29) can be written as

$$
L_{0}=\frac{1}{2} \rho_{0} u^{2}+f_{0} \psi_{i}^{j}\left(\bar{\sigma}_{j}^{i}+\bar{p} \delta_{j}^{i}\right) .
$$

Since the coefficient of viscosity $\mu_{0}$ of the $\Omega(0)$ substratum may be very small, we introduce the following assumption. 
Assumption 2 In the nonrelativistic limit, i.e., $u / c \ll 1$, where $u=|\boldsymbol{u}|$, $\boldsymbol{u}$ is the velocity of the $\Omega(0)$ substratum, we suppose that $\mu_{0} \approx 0$ and we have the following conditions

$$
\psi_{i j} \approx 0, \quad \partial_{\mu} \psi_{i j} \approx 0, \quad \partial_{\mu} \partial_{\nu} \psi_{i j} \approx 0,
$$

where

$$
\partial_{\mu} \equiv\left(\frac{\partial}{\partial x^{0}}, \frac{\partial}{\partial x^{1}}, \frac{\partial}{\partial x^{2}}, \frac{\partial}{\partial x^{3}}\right) .
$$

According to the Stokes-Helmholtz resolution theorem, refers to, for instance, [52, 53], every sufficiently smooth vector field can be decomposed into irrotational and solenoidal parts. Thus, there exists a scalar function $\psi$ and a vector function $\mathbf{R}$ such that the velocity field $\mathbf{u}$ of the $\Omega(0)$ substratum can be represented by [52, 53]

$$
\mathbf{u}=\nabla \varphi+\nabla \times \mathbf{R}
$$

where $\nabla \times \varphi=0, \nabla \cdot \mathbf{R}=0$.

We introduce the following definition of a vector function $\vec{\xi}$

$$
\frac{\partial \vec{\xi}}{\partial(c t)}=\nabla \times \mathbf{R}
$$

Putting Eq.(35) into Eq.(34), we have

$$
\mathbf{u}=\nabla \varphi+\frac{\partial \vec{\xi}}{\partial(c t)}
$$

Based on Assumption 2 and Eq.(36), Eq.(31) can be written as

$$
L_{0}=\frac{\rho_{0}}{2}\left[(\nabla \varphi)^{2}+2(\nabla \varphi) \cdot \frac{\partial \vec{\xi}}{\partial(c t)}+\left(\frac{\partial \vec{\xi}}{\partial(c t)}\right)^{2}\right] .
$$

Using Eq.(10), Eq.(37) can be written as

$$
L_{0}=\frac{m_{0}^{2}}{2 \rho_{0} q_{0}^{2}}(\nabla \Phi)^{2}+\frac{m_{0}}{q_{0}}(\nabla \Phi) \cdot \frac{\partial \vec{\xi}}{\partial(c t)}+\frac{\rho_{0}}{2}\left(\frac{\partial \vec{\xi}}{\partial(c t)}\right)^{2} .
$$

We introduce the following definitions

$$
\begin{aligned}
& \psi_{00}=-a_{00} \Phi, \quad \psi_{0 i}=\psi_{i 0}=a_{0 i} \xi_{i} \\
& \vec{\psi}_{0}=\psi_{01} \mathbf{i}+\psi_{02} \mathbf{j}+\psi_{03} \mathbf{k} .
\end{aligned}
$$

where $a_{00}>0$ and $a_{0 i}>0$ are 4 parameters to be determined, $i=1,2,3$.

Eq.(39) and Eq.(30) have defined a rank 2 symmetric tensor $\psi_{\mu \nu}$. We require that for some special values of $a_{00}$ and $a_{0 i}$, Eq.(38) can be written as

$$
\begin{aligned}
L_{0} & =\left(\nabla \psi_{00}\right)^{2}-2\left(\nabla \psi_{00}\right) \cdot \frac{\partial \vec{\psi}_{0}}{\partial(c t)}+\left(\frac{\partial \vec{\psi}_{0}}{\partial(c t)}\right)^{2} \\
& =\left(\nabla \psi_{00}-\frac{\partial \vec{\psi}_{0}}{\partial(c t)}\right)^{2} .
\end{aligned}
$$

Comparing Eq.(41) and Eq.(38) and using Eqs.(39-40), we have

$$
a_{00}=\sqrt{\frac{m_{0}^{2}}{2 \rho_{0} q_{0}^{2}}}, \quad a_{0 i}=\sqrt{\frac{\rho_{0}}{2}} .
$$

In order to construct the Lagrangian $L_{0}$ described in Eq.(41) based on the tensorial potential $\psi_{\mu \nu}$, we should consider all the possible products of derivatives of the tensor $\psi_{\mu \nu}$. If we require that the two tensor indices of $\psi_{\mu \nu}$ 
are different from each other and the two tensor indices of $\psi_{\mu \nu}$ are different from the derivative index, we have the following two possible products ([10], p43):

$$
L_{1}=\partial_{\sigma} \psi_{\mu \nu} \partial^{\sigma} \psi^{\mu \nu}, \quad L_{2}=\partial_{\sigma} \psi_{\mu \nu} \partial^{\mu} \psi^{\sigma \nu}
$$

If there are two indices of $\psi_{\mu \nu}$ which are equal, or one of the indices of $\psi_{\mu \nu}$ is the same as the derivative index, we may have the following three possible products ([10], p43):

$$
\begin{aligned}
& L_{3}=\partial_{\nu} \psi_{\mu \nu} \partial_{\sigma} \psi_{\mu}^{\sigma}, \quad L_{4}=\partial^{\mu} \psi_{\mu \nu} \partial^{\nu} \psi, \\
& L_{5}=\partial_{\lambda} \psi \partial^{\lambda} \psi .
\end{aligned}
$$

where $\psi$ is the trace of $\psi_{\mu \nu}$, i.e., $\psi \equiv \psi_{\lambda}^{\lambda}=\eta_{\alpha \beta} \psi^{\alpha \beta}$,

$$
\partial^{\mu} \equiv \eta^{\mu \nu} \partial_{\nu}=\left(\frac{\partial}{\partial x^{0}},-\frac{\partial}{\partial x^{1}},-\frac{\partial}{\partial x^{2}},-\frac{\partial}{\partial x^{3}}\right) .
$$

$L_{3}$ may be omitted because it can be converted to $L_{2}$ by integration by parts ([10], p43). Inspired by W. Thirring [34] and R. P. Feynman ([10], p43), we introduce the following assumption.

Assumption 3 The Lagrangian $L_{0}$ for free fields of the $\Omega(0)$ substratum can be written as

$$
L_{0}=c_{1} L_{1}+c_{2} L_{2}+c_{4} L_{4}+c_{5} L_{5}+L_{\text {more }},
$$

where $c_{i}, i=1,2,4,5$ are 4 parameters to be determined, $L_{\text {more }}$ denotes those terms involving more than two derivatives of $\psi_{\mu \nu}$.

Proposition 4 Suppose that Assumption 2 is valid and we have the following conditions

$$
\frac{\partial \psi_{00}}{\partial(c t)} \approx 0, \quad \frac{\partial \psi_{0 i}}{\partial x^{j}} \approx 0
$$

If we set

$$
c_{1}=\frac{1}{2}, \quad c_{2}=-2, \quad c_{4}=-6, \quad c_{5}=-\frac{3}{2},
$$

then we have

$$
c_{1} L_{1}+c_{2} L_{2}+c_{4} L_{4}+c_{5} L_{5} \approx\left(\nabla \psi_{00}-\frac{\partial \vec{\psi}_{0}}{\partial(c t)}\right)^{2} .
$$

Proof of Proposition 4. Based on Eq.(32) and Eq.(48) and noticing $\psi^{00}=\psi_{00}, \psi^{0 i}=-\psi_{0 i}$, we have

$$
L_{1} \approx-\left(\nabla \psi_{00}\right)^{2}-2\left(\frac{\partial \vec{\psi}_{0}}{\partial(c t)}\right)^{2}
$$

Using Eq.(32) and Eq.(48) and noticing $\psi^{0 i}=-\psi_{0 i}$, we have

$$
L_{2} \approx-2\left(\nabla \psi_{00}\right) \cdot \frac{\partial \vec{\psi}_{0}}{\partial(c t)}-\left(\frac{\partial \vec{\psi}_{0}}{\partial(c t)}\right)^{2} .
$$

Similarly, we have the following results

$$
\begin{aligned}
& L_{3} \approx\left(\nabla \psi_{00}\right) \cdot \frac{\partial \vec{\psi}_{0}}{\partial(c t)}, \\
& L_{4} \approx-\left(\nabla \psi_{00}\right)^{2} .
\end{aligned}
$$

Using Eqs.(51-54), we obtain Eq.(50). Proof ends. 


\section{INTERACTION TERMS OF THE LAGRANGIAN OF A SYSTEM OF THE $\Omega(0)$ SUBSTRATUM, THE $\Omega(1)$ SUBSTRATUM AND MATTER}

In order to derive the field equation, we should explore the possible interaction terms of the Lagrangian of a system of the $\Omega(0)$ substratum, the $\Omega(1)$ substratum and matter. If we suppose that the $\Omega(0)$ substratum is an idea fluid, then from Eq.(34) we have $\mathbf{u}=\nabla \varphi$. Ignoring the damping force $\rho_{0} Q \mathbf{v}_{s}$ in Eq.(4) and using $\mathbf{u}=\nabla \varphi$, Eq.(4) can be written as

$$
\mathbf{F}_{Q}=-\rho_{0} Q \nabla \varphi
$$

A particle is modelled as a point sink of the $\Omega(0)$ substratum $[38,47,48]$. Thus, the interaction term of the Lagrangian of a system of the $\Omega(0)$ substratum and a particle can be written as

$$
L_{\text {int }}=\rho_{0} Q \varphi
$$

Thus, the interaction term of the Lagrangian of a system of the $\Omega(0)$ substratum and continuously distributed particles can be written as

$$
L_{\text {int }}=\rho_{0} \rho_{s} \varphi
$$

Putting Eq.(10) into Eq.(57), we have

$$
L_{i n t}=-\rho_{m} \Phi .
$$

The 00 term of the energy-momentum tensor $T_{\mu \nu}^{m}$ of a particle is $T_{m}^{00}=\rho_{m} c^{2}$. Thus, using Eq.(39), Eq.(58) can be written as

$$
L_{i n t}=f_{0} \psi_{00} T_{m}^{00},
$$

where

$$
f_{0}=\frac{1}{a_{00} c^{2}}
$$

From Eq.(60), Eq.(42) and Eq.(6), we have

$$
f_{0}=\sqrt{\frac{2 \rho_{0} q_{0}^{2}}{m_{0}^{2} c^{4}}}=\sqrt{\frac{8 \pi \gamma_{N}}{c^{4}}}, \quad \frac{1}{a_{00}^{2}}=8 \pi \gamma_{N} .
$$

Inspired by Eq.(59) and Eq.(31), we introduce the following assumption.

Assumption 5 The interaction terms of the Lagrangian of a system of the $\Omega(0)$ substratum, the $\Omega(1)$ substratum and matter can be written as the following form.

$$
L_{i n t}=f_{0} \psi_{\mu \nu} T_{m}^{\mu \nu}+f_{0} \psi_{\mu \nu} T_{\Omega(1)}^{\mu \nu}+O\left[\left(f_{0} \psi_{\mu \nu}\right)^{2}\right]
$$

where $T_{m}^{\mu \nu}$ and $T_{\Omega(1)}^{\mu \nu}$ are the contravariant energy-momentum tensor of the system of the matter and the $\Omega(1)$ substratum respectively, $O\left[\left(f_{0} \psi_{\mu \nu}\right)^{2}\right]$ denotes those terms which are small quantities of the order of $\left(f_{0} \psi_{\mu \nu}\right)^{2}$.

\section{DERIVATION OF THE FIELD EQUATION}

Based on Proposition 4 and Assumption 5, the total Lagrangian $L_{t o t}$ of a system of the $\Omega(0)$ substratum, the $\Omega(1)$ substratum and matter can be written as

$$
\begin{aligned}
L_{t o t}= & \frac{1}{2} \partial_{\lambda} \psi_{\mu \nu} \partial^{\lambda} \psi^{\mu \nu}-2 \partial_{\lambda} \psi_{\mu \nu} \partial^{\mu} \psi^{\lambda \nu}-6 \partial^{\mu} \psi_{\mu \nu} \partial^{\nu} \psi \\
& -\frac{3}{2} \partial_{\lambda} \psi \partial^{\lambda} \psi+L_{\text {more }}+f_{0} \psi_{\mu \nu}\left(T_{m}^{\mu \nu}+T_{\Omega(1)}^{\mu \nu}\right) \\
& +O\left[\left(f_{0} \psi_{\mu \nu}\right)^{2}\right] .
\end{aligned}
$$


Theorem 6 If we ignore those terms which are small quantities of the order of $\left(f_{0} \psi_{\mu \nu}\right)^{2}$ and those terms involving more than two derivatives of $\psi_{\mu \nu}$ in Eq.(63), i.e., $O\left[\left(f_{0} \psi_{\mu \nu}\right)^{2}\right]$ and $L_{m o r e}$, then the field equation for the total Lagrangian $L_{\text {tot }}$ in Eq.(63) can be written as

$$
\begin{aligned}
& \partial_{\sigma} \partial^{\sigma} \psi_{\alpha \beta}-2\left(\partial^{\sigma} \partial_{\alpha} \psi_{\beta \sigma}+\partial^{\sigma} \partial_{\beta} \psi_{\alpha \sigma}\right)-6\left(\eta_{\alpha \beta} \partial_{\sigma} \partial_{\lambda} \psi^{\sigma \lambda}\right. \\
& \left.+6 \partial_{\alpha} \partial_{\beta} \psi\right)-3 \eta_{\alpha \beta} \partial_{\sigma} \partial^{\sigma} \psi=f_{0}\left(T_{\mu \nu}^{m}+T_{\mu \nu}^{\Omega(1)}\right) .
\end{aligned}
$$

Proof of Theorem 6. Starting from the Lagrangian in Eq.(63), we have the following Euler-Lagrange equation [37]

$$
\frac{\partial L_{t o t}}{\partial \psi^{\alpha \beta}}-\frac{\partial}{\partial x^{\sigma}}\left(\frac{\partial L_{t o t}}{\partial\left(\partial_{\sigma} \psi^{\alpha \beta}\right)}\right)=0
$$

We can verify the following results $([10], \mathrm{p} 43 ;[34])$

$$
\begin{aligned}
\frac{\partial}{\partial x^{\sigma}}\left[\frac{\partial\left(\partial_{\sigma} \psi_{\mu \nu} \partial^{\sigma} \psi^{\mu \nu}\right)}{\partial\left(\partial_{\sigma} \psi^{\alpha \beta}\right)}\right] & =2 \partial_{\sigma} \partial^{\sigma} \psi_{\alpha \beta}, \\
\frac{\partial}{\partial x^{\sigma}}\left[\frac{\partial\left(\partial_{\sigma} \psi_{\mu \nu} \partial^{\mu} \psi^{\sigma \nu}\right)}{\partial\left(\partial_{\sigma} \psi^{\alpha \beta}\right)}\right] & =\partial^{\sigma} \partial_{\alpha} \psi_{\beta \sigma}+\partial^{\sigma} \partial_{\beta} \psi_{\alpha \sigma}, \\
\frac{\partial}{\partial x^{\sigma}}\left[\frac{\partial\left(\partial^{\mu} \psi_{\mu \nu} \partial^{\nu} \psi\right)}{\partial\left(\partial_{\sigma} \psi^{\alpha \beta}\right)}\right] & =\partial_{\alpha} \partial_{\beta} \psi+\eta_{\alpha \beta} \partial_{\sigma} \partial_{\lambda} \psi^{\sigma \lambda}, \\
\frac{\partial}{\partial x^{\sigma}}\left[\frac{\partial\left(\partial_{\sigma} \psi \partial^{\sigma} \psi\right)}{\partial\left(\partial_{\sigma} \psi^{\alpha \beta}\right)}\right] & =2 \eta_{\alpha \beta} \partial_{\sigma} \partial^{\sigma} \psi \\
\frac{\partial L_{t o t}}{\partial \psi^{\alpha \beta}} & =f_{0}\left(T_{\alpha \beta}^{m}+T_{\alpha \beta}^{\Omega(1)}\right) .
\end{aligned}
$$

Putting Eq.(63) into Eq.(65) and using Eqs.(66-70), we obtain Eq.(64). Proof ends.

We introduce the following notation

$$
\begin{aligned}
\Psi^{\mu \nu}= & \partial_{\lambda} \partial^{\lambda} \psi^{\mu \nu}-2 \partial_{\lambda} \partial^{\mu} \psi^{\nu \lambda}-2 \partial_{\lambda} \partial^{\nu} \psi^{\mu \lambda} \\
& -6 \eta^{\mu \nu} \partial_{\sigma} \partial_{\lambda} \psi^{\sigma \lambda}-6 \partial^{\mu} \partial^{\nu} \psi-3 \eta^{\mu \nu} \partial_{\lambda} \partial^{\lambda} \psi
\end{aligned}
$$

Thus, Eq.(64) can be written as

$$
\Psi^{\mu \nu}=f_{0}\left(T_{m}^{\mu \nu}+T_{\Omega(1)}^{\mu \nu}\right) .
$$

We introduce the following definition of total energy-momentum tensor of the system of the matter, the $\Omega(1)$ substratum and the $\Omega(0)$ substratum

$$
T^{\mu \nu}=T_{m}^{\mu \nu}+T_{\Omega(1)}^{\mu \nu}+T_{\Omega(0)}^{\mu \nu},
$$

where $T_{\Omega(0)}^{\mu \nu}$ is the energy-momentum tensor of the $\Omega(0)$ substratum.

Adding the term $f_{0} T_{\Omega(0)}^{\mu \nu}$ on both sides of Eq.(72) and using Eq.(73), we have

$$
\Psi^{\mu \nu}+f_{0} T_{\Omega(0)}^{\mu \nu}=f_{0} T^{\mu \nu} .
$$

For the total system of matter, the $\Omega(1)$ substratum and the $\Omega(0)$ substratum, the law of conservation of energy and momentum is ([1], p163; [49], p155)

$$
\partial_{\mu} T^{\mu \nu}=0
$$

Comparing Eq.(75) and Eq.(74), we have

$$
\partial_{\mu}\left(\Psi^{\mu \nu}+f_{0} T_{\Omega(0)}^{\mu \nu}\right)=0 .
$$

Noticing Eqs.(66-70), we introduce the following notation ([10], p43)

$$
\begin{aligned}
& H^{\mu \nu}=f_{1} \partial_{\lambda} \partial^{\lambda} \psi^{\mu \nu}+f_{2}\left(\partial_{\lambda} \partial^{\mu} \psi^{\nu \lambda}+\partial_{\lambda} \partial^{\nu} \psi^{\mu \lambda}\right) \\
& +f_{3}\left(\partial^{\mu} \partial^{\nu} \psi+\eta^{\mu \nu} \partial_{\sigma} \partial_{\lambda} \psi^{\sigma \lambda}\right)+f_{4} \eta^{\mu \nu} \partial_{\lambda} \partial^{\lambda} \psi,
\end{aligned}
$$


where $f_{i}, i=1,2,3,4$ are 4 arbitrary parameters.

If we require that

$$
\partial_{\mu} H^{\mu \nu}=0
$$

then, we can verify the following relationships ([10], p44; [34])

$$
\begin{aligned}
& f_{1}+f_{2}=0, \\
& f_{2}+f_{3}=0, \\
& f_{3}+f_{4}=0 .
\end{aligned}
$$

We choose $f_{1}=1, f_{2}=-1, f_{3}=1, f_{4}=-1$ in Eq.(77) and introduce the following notation

$$
\begin{aligned}
\Theta^{\mu \nu}= & \partial_{\lambda} \partial^{\lambda} \psi^{\mu \nu}-\left(\partial_{\lambda} \partial^{\mu} \psi^{\nu \lambda}+\partial_{\lambda} \partial^{\nu} \psi^{\mu \lambda}\right) \\
& +\left(\partial^{\mu} \partial^{\nu} \psi+\eta^{\mu \nu} \partial_{\sigma} \partial_{\lambda} \psi^{\sigma \lambda}\right)-\eta^{\mu \nu} \partial_{\lambda} \partial^{\lambda} \psi .
\end{aligned}
$$

We can verify the following result ([10], p44; [34])

$$
\partial_{\mu} \Theta^{\mu \nu}=0 .
$$

From Eq.(83) and Eq.(76), we have

$$
\partial_{\mu}\left(\Psi^{\mu \nu}+f_{0} T_{\Omega(0)}^{\mu \nu}-b_{0} \Theta^{\mu \nu}\right)=0
$$

where $b_{0}$ is an arbitrary parameter.

We introduce the following definition of a tensor $\omega^{\mu \nu}$

$$
f_{0} \omega^{\mu \nu}=\Psi^{\mu \nu}+f_{0} T_{\Omega(0)}^{\mu \nu}-b_{0} \Theta^{\mu \nu},
$$

where $b_{0}$ is a parameter to be determined.

Assumption 7 In the nonrelativistic limit, i.e., $u / c \ll 1$, where $u=|\boldsymbol{u}|, \boldsymbol{u}$ is the velocity of the $\Omega(0)$ substratum, the following relationship is valid

$$
T^{\mu \nu}-\omega^{\mu \nu} \approx T_{m}^{\mu \nu}
$$

Theorem 8 Suppose that Assumption 7 is valid, then, the field equation (64) can be written as

$$
\begin{aligned}
& \partial_{\lambda} \partial^{\lambda} \psi^{\mu \nu}-\partial_{\lambda} \partial^{\mu} \psi^{\nu \lambda}-\partial_{\lambda} \partial^{\nu} \psi^{\mu \lambda}+\partial^{\mu} \partial^{\nu} \psi \\
& +\eta^{\mu \nu} \partial_{\sigma} \partial_{\lambda} \psi^{\sigma \lambda}-\eta^{\mu \nu} \partial_{\lambda} \partial^{\lambda} \psi=-f_{0}\left(T^{\mu \nu}-\omega^{\mu \nu}\right) .
\end{aligned}
$$

Proof of Theorem 8. From Eq.(85) and Eq.(74), we have the following field equation

$$
\Theta^{\mu \nu}=\frac{f_{0}}{b_{0}}\left(T^{\mu \nu}-\omega^{\mu \nu}\right)
$$

Now our task is to determine the parameter $b_{0}$ in the field equation (88). A natural idea is that the 00 component of the field equation (88) reduces to the field equation Eq.(11) in the nonrelativistic limit. Noticing Eq.(82), the 00 component of the field equation (88) is

$$
\begin{aligned}
\partial_{\lambda} \partial^{\lambda} \psi^{00} & -2 \partial_{\lambda} \partial^{0} \psi^{0 \lambda}+\partial^{0} \partial^{0} \psi \\
& +\partial_{\sigma} \partial_{\lambda} \psi^{\sigma \lambda}-\partial_{\lambda} \partial^{\lambda} \psi=\frac{f_{0}}{b_{0}}\left(T^{00}-\omega^{00}\right) .
\end{aligned}
$$

Take the trace of the field equation Eq.(88), we have

$$
\partial_{\sigma} \partial_{\lambda} \psi^{\sigma \lambda}-\partial_{\lambda} \partial^{\lambda} \psi=\frac{f_{0}}{2 b_{0}}(T-\omega)
$$

where $T$ and $\omega$ are the traces of $T^{\mu \nu}$ and $\omega^{\mu \nu}$ respectively, i.e., $T \equiv T_{\lambda}^{\lambda}=\eta_{\alpha \beta} T^{\alpha \beta}, \omega \equiv \omega_{\lambda}^{\lambda}=\eta_{\alpha \beta} \omega^{\alpha \beta}$. 
Subtracting Eq.(90) from Eq.(89), we have

$$
\begin{aligned}
\partial_{\lambda} \partial^{\lambda} \psi^{00} & -2 \partial_{\lambda} \partial^{0} \psi^{0 \lambda}+\partial^{0} \partial^{0} \psi \\
& =\frac{f_{0}}{b_{0}}\left(T^{00}-\frac{T}{2}-\omega^{00}+\frac{\omega}{2}\right) .
\end{aligned}
$$

If the field is time-independent, then Eq.(91) reduces to

$$
-\nabla^{2} \psi^{00}=\frac{f_{0}}{b_{0}}\left(T^{00}-\frac{T}{2}-\omega^{00}+\frac{\omega}{2}\right) .
$$

According to Eq.(86), in the nonrelativistic limit, we have

$$
T^{00}-\omega^{00} \approx T_{m}^{00}=\rho_{m} c^{2}, \quad T-\omega \approx T_{m} \approx \rho_{m} c^{2},
$$

where $T_{m}$ is the trace of $T_{m}^{\mu \nu}$, i.e., $T_{m} \equiv \eta_{\alpha \beta} T_{m}^{\alpha \beta}$.

Using Eq.(39), Eq.(42), Eq.(61) and Eq.(93), Eq.(92) can be written as

$$
\nabla^{2} \Phi=\frac{1}{b_{0}} 4 \pi \gamma_{N} \rho_{m}
$$

Comparing Eq.(94) and Eq.(11), we obtain $b_{0}=-1$. Therefore, using Eq.(82) and $b_{0}=-1$, the field equation Eq.(88) can be written as Eq.(87). Proof ends.

We can verify that the field equation Eq.(87) is invariant under the gauge transformation ([10], p45; [34])

$$
\psi^{\mu \nu} \rightarrow \psi^{\mu \nu}+\partial^{\mu} \Lambda^{\nu}+\partial^{\nu} \Lambda^{\mu},
$$

where $\Lambda^{\mu}$ is an arbitrary vector field.

We introduce the following definition

$$
\phi^{\mu \nu}=\psi^{\mu \nu}-\frac{1}{2} \eta^{\mu \nu} \psi
$$

Using Eq.(96), the field equation (87) can be written as

$$
\begin{aligned}
\partial_{\lambda} \partial^{\lambda} \phi^{\mu \nu} & -\partial_{\lambda} \partial^{\mu} \phi^{\nu \lambda}-\partial_{\lambda} \partial^{\nu} \phi^{\mu \lambda} \\
& +\eta^{\mu \nu} \partial_{\sigma} \partial_{\lambda} \phi^{\sigma \lambda}=-f_{0} T^{\mu \nu} .
\end{aligned}
$$

We introduce the following Hilbert gauge condition [34]

$$
\partial_{\mu}\left(\psi^{\mu \nu}-\frac{1}{2} \eta^{\mu \nu} \psi\right)=0
$$

Using Eq.(96), the Hilbert gauge condition Eq.(98) simplifies to

$$
\partial_{\mu} \phi^{\mu \nu}=0 .
$$

Applying Eq.(99) in Eq.(97), we obtain the following proposition [34].

Proposition 9 If we impose the Hilbert gauge condition Eq.(98) on the fields, then, the field equation Eq.(87) simplifies to

$$
\partial_{\lambda} \partial^{\lambda}\left(\psi^{\mu \nu}-\frac{1}{2} \eta^{\mu \nu} \psi\right)=-f_{0}\left(T^{\mu \nu}-\omega^{\mu \nu}\right)
$$

If the tensor potential $\psi^{\mu \nu}$ does not satisfy the Hilbert gauge condition Eq.(98), then we can always construct a new tensor potential $\bar{\psi}^{\mu \nu}$ by the following gauge transformation [34]

$$
\bar{\psi}^{\mu \nu}=\psi^{\mu \nu}+\partial^{\mu} \Lambda^{\nu}+\partial^{\nu} \Lambda^{\mu}
$$

such that the new tensor potential $\bar{\psi}^{\mu \nu}$ does satisfy the Hilbert gauge condition Eq.(98).

Using Eq.(96), the field equation Eq.(100) can be written as

$$
\partial_{\lambda} \partial^{\lambda} \phi^{\mu \nu}=-f_{0}\left(T^{\mu \nu}-\omega^{\mu \nu}\right) .
$$

The field equation Eq.(102) can also be written as

$$
\eta^{\alpha \beta} \frac{\partial^{2} \phi^{\mu \nu}}{\partial x^{\alpha} \partial x^{\beta}}=-f_{0}\left(T^{\mu \nu}-\omega^{\mu \nu}\right)
$$

We noticed that the tensorial field equation Eq.(103) is similar to the wave equation of electromagnetic fields. 


\section{CONSTRUCTION OF A TENSORIAL POTENTIAL IN INERTIAL COORDINATE SYSTEMS}

The existence of the $\Omega(1)$ substratum allows us to introduce the following definition of inertia coordinates system.

Definition 10 If a coordinates system $S$ is static or moving with a constant velocity relative to the reference frame $S_{\Omega(1)}$, then, we call such a coordinates system as an inertia coordinates system.

The field equation Eq.(97) and Eq.(100) are valid in the reference frame $S_{\Omega(1)}$. We will explore the possibility of constructing a tensorial potential in an arbitrary inertial system $S^{\prime}$. In an inertial coordinate system $S$, an arbitrary event is characterized by the four space-time coordinates $(t, x, y, z)$. In an inertial system $S^{\prime}$, this event is characterized by four other coordinates $\left(t^{\prime}, x^{\prime}, y^{\prime}, z^{\prime}\right)$. We assume that the origins of the Cartesian coordinates in the two inertial systems $S$ and $S^{\prime}$ coincide at the time $t=t^{\prime}=0$. Then, the connections between these space-time coordinates are given by a homogeneous linear transformation keeping the quantity $s^{2}=c t^{2}-x^{2}-y^{2}-z^{2}$ invariant, i.e., ([1], p92)

$$
s^{2}=c^{2} t^{2}-x^{2}-y^{2}-z^{2}=c^{2} t^{\prime 2}-x^{\prime 2}-y^{\prime 2}-z^{\prime 2}=s^{\prime 2},
$$

We introduce the following two coordinate systems

$$
\begin{aligned}
& x^{0}=c t, x^{1}=x, x^{2}=y, x^{3}=z, \\
& x^{\prime 0}=c t^{\prime}, x^{\prime 1}=x^{\prime}, x^{\prime 2}=y^{\prime}, x^{\prime 3}=z^{\prime} .
\end{aligned}
$$

The homogeneous linear transformation keeping the quantity $s^{2}$ invariant, which is usually called the Lorentz transformation, can be written as ([54], p57; [1], p92)

$$
x^{\prime \mu}=\alpha_{\nu}^{\mu} x^{\nu}
$$

where $\alpha^{\mu}{ }_{\nu}$ are coefficients depend only on the angles between the spatial axes in the two inertial systems $S$ and $S^{\prime}$ and on the relative velocity of $S$ and $S^{\prime}, \mu, \nu=0,1,2,3$.

Proposition 11 Suppose that the field equation Eq.(102) is valid in the the reference frame $S_{\Omega(1)}$. Then, in an arbitrary inertial system $S^{\prime}$, there exists a symmetric tensor $\phi_{\mu \nu}^{\prime}$ satisfies the following wave equation

$$
\partial_{\lambda}^{\prime} \partial^{\prime \lambda} \phi^{\prime \mu \nu}=f_{0}\left(T^{\prime \mu \nu}-\omega^{\prime \mu \nu}\right)
$$

where $T^{\prime \mu \nu}$ and $\omega^{\prime \mu \nu}$ are corresponding tensors of $T^{\mu \nu}$ and $\omega^{\mu \nu}$ in the arbitrary inertial coordinate system $S^{\prime}$ respectively.

Proof of Proposition 11. According to the definition of a tensor of second rank in spacetime, we have ([1], p108)

$$
\begin{aligned}
\phi^{\prime \mu \nu} & =\alpha^{\mu}{ }_{\sigma} \alpha_{\lambda}^{\nu} \phi^{\sigma \lambda}, \\
\left(T^{\prime \mu \nu}-\omega^{\prime \mu \nu}\right) & =\alpha^{\mu}{ }_{\sigma} \alpha^{\nu}{ }_{\lambda}\left(T^{\mu \nu}-\omega^{\mu \nu}\right) .
\end{aligned}
$$

Eq.(108) and Eq.(109) can also be written as ([1], p108)

$$
\begin{aligned}
\phi^{\mu \nu} & =\phi^{\prime \sigma \lambda} \alpha_{\sigma}^{\mu} \alpha_{\lambda}^{\nu}, \\
\left(T^{\mu \nu}-\omega^{\mu \nu}\right) & =\left(T^{\prime \sigma \lambda}-\omega^{\prime \sigma \lambda}\right) \alpha^{\mu}{ }_{\sigma} \alpha_{\lambda}^{\nu} .
\end{aligned}
$$

Putting Eq.(110) and Eq.(111) into Eq.(102), we have

$$
\partial_{\lambda} \partial^{\lambda}\left(\phi^{\prime \sigma \lambda} \alpha_{\sigma}^{\mu} \alpha_{\lambda}^{\nu}\right)=f_{0}\left[\left(T^{\prime \sigma \lambda}-\omega^{\prime \sigma \lambda}\right) \alpha_{\sigma}^{\mu} \alpha_{\lambda}^{\nu}\right]
$$

Eq.(112) can be written as

$$
\left[\partial_{\lambda} \partial^{\lambda} \phi_{\sigma \lambda}^{\prime}-f_{0}\left(T^{\prime \sigma \lambda}-\omega^{\prime \sigma \lambda}\right)\right] \alpha_{\sigma}^{\mu} \alpha_{\lambda}^{\nu}=0
$$

Since $\alpha^{\mu}{ }_{\nu}$ are coefficients of an arbitrary Lorentz transformation, we obtain

$$
\partial_{\lambda} \partial^{\lambda} \phi^{\prime \mu \nu}=f_{0}\left(T^{\prime \mu \nu}-\omega^{\prime \mu \nu}\right)
$$

Eq.(114) can be written as Eq.(107). Proof ends.

Proposition 12 Suppose that the field equation Eq.(97) is valid in the the reference frame $S_{\Omega(1)}$. Then, in an arbitrary inertial system $S^{\prime}$, there exists a symmetric tensor $\phi_{\mu \nu}^{\prime}$ satisfies the following field equation

$$
\begin{aligned}
\partial_{\lambda}^{\prime} \partial^{\prime \lambda} \phi^{\prime \mu \nu} & -\partial_{\lambda}^{\prime} \partial^{\prime \mu} \phi^{\prime \nu \lambda}-\partial_{\lambda}^{\prime} \partial^{\prime \nu} \phi^{\prime \mu \lambda} \\
& +\eta^{\mu \nu} \partial_{\sigma}^{\prime} \partial_{\lambda}^{\prime} \phi^{\prime \sigma \lambda}=-f_{0}\left(T^{\prime \mu \nu}-\omega^{\prime \mu \nu}\right) .
\end{aligned}
$$

The Proof of Proposition 12 is similar to the Proof of Proposition 11. 


\section{THE EQUATION OF MOTION OF A POINT PARTICLE IN A GRAVITATIONAL FIELD AND INTRODUCTION OF AN EFFECTIVE RIEMANNIAN SPACETIME}

In this section, we study the equation of motion of a free point particle in a gravitational field. The Lagrangian of a free point particle can be written as $([10], \mathrm{p} 57 ;[34])$

$$
L_{0}=\frac{1}{2} m \frac{d x^{\mu}}{d \tau} \frac{d x^{\nu}}{d \tau}=\frac{1}{2} m u^{\mu} u_{\mu}=\frac{1}{2} m \eta_{\mu \nu} u^{\mu} u^{\nu}
$$

where $m$ is the rest mass of the point particle, $\tau \equiv \sqrt{d x^{\mu} d x_{\mu}}$ is the proper time interval, $u^{\mu} \equiv d x^{\mu} / d \tau$.

Suppose that $T_{\Omega(1)}^{\mu \nu} \approx 0$. Ignoring those higher terms $O\left[\left(f_{0} \psi_{\mu \nu}\right)^{2}\right]$ in Eq. $(62)$, the interaction term of the Lagrangian of a system of the $\Omega(0)$ substratum, the $\Omega(1)$ substratum and the point particle can be written as the following form $([10], \mathrm{p} 57 ;[34])$

$$
L_{i n t}=f_{0} \psi_{\mu \nu} m u^{\mu} u^{\nu}
$$

Using Eq.(117) and Eq.(116), the total Lagrangian $L_{\text {tot }}$ of a system of the $\Omega(0)$ substratum, the $\Omega(1)$ substratum and the point particle can be written as ([10], p57)

$$
L_{t o t}=L_{0}+L_{i n t}=\frac{1}{2} m u^{\mu} u_{\mu}+f_{0} \psi_{\mu \nu} m u^{\mu} u^{\nu}
$$

The Euler-Lagrange equation for the the total Lagrangian $L_{t o t}$ can be written as ([54],p111)

$$
\frac{d}{d \tau}\left[\left(\eta_{\mu \nu}+2 f_{0} \psi_{\mu \nu}\right) \frac{d x^{\nu}}{d \tau}\right]-f_{0} \frac{\partial \psi_{\alpha \beta}}{\partial x^{\mu}} \frac{d x^{\alpha}}{d \tau} \frac{d x^{\beta}}{d \tau}=0
$$

We notice that the equation of motion (119) of a point particle in gravitational field is similar to the equation of a geodesic line (121) in a Riemannian spacetime. Thus, it is natural for us to introduce the following definition of a metric tensor $g_{\mu \nu}$ of a Riemannian spacetime ([10], p57)

$$
g_{\mu \nu}=\eta_{\mu \nu}+2 f_{0} \psi_{\mu \nu}
$$

Then, the equation of motion Eq.(119) can be approximately written as ([10], p58)

$$
\frac{d}{d \tau_{g}}\left(g_{\mu \nu} \frac{d x^{\nu}}{d \tau_{g}}\right)=\frac{1}{2} \frac{\partial g_{\alpha \beta}}{\partial x^{\mu}} \frac{d x^{\alpha}}{d \tau_{g}} \frac{d x^{\beta}}{d \tau_{g}}
$$

where $\tau_{g}$ is the proper time interval in the Riemannian spacetime with a metric tensor $g_{\mu \nu}$.

Eq.(121) is a geodesic line in a Riemannian spacetime with a metric tensor $g_{\mu \nu}$, which can also be written as ([55], p51)

$$
\frac{d^{2} x^{\mu}}{d \tau_{g}^{2}}+\Gamma_{\nu \sigma}^{\mu} \frac{d x^{\nu}}{d \tau_{g}} \frac{d x^{\sigma}}{d \tau_{g}}=0
$$

where $\Gamma_{\nu \sigma}^{\mu}$ is the Christoffel symbol.

Thus, we find that the equation of motion (119) of a point particle in gravitational field is approximately a geodesic line described in Eq.(122) in a Riemannian spacetime with a metric tensor $g_{\mu \nu}$.

According to Assumption 1, the particles that constitute the $\Omega(1)$ substratum are sinks in the $\Omega(0)$ substratum. Thus, the movements of the $\Omega(1)$ substratum in gravitational field will be different from the Maxwell's equation. We notice that the equation (121) of motion of a point particle in gravitational field is a generalization of the equation of motion of a point particle in vacuum free of gravitational field. The law of propagation of an electromagnetic wave front in vacuum free of gravitational field is Eq.(16). Therefore, it is natural for us to speculate that the law of propagation of an electromagnetic wave front in gravitational field is a generalization of Eq.(16). Thus, we introduce the following assumption.

Assumption 13 To first order of $f_{0}$, the law of propagation of an electromagnetic wave front $\omega\left(x_{0}, x_{1}, x_{2}, x_{3}\right)$ in gravitational field is

$$
g_{\mu \nu} \frac{\partial \omega}{\partial x^{\mu}} \frac{\partial \omega}{\partial x^{\nu}}=0
$$

where $\omega\left(x_{0}, x_{1}, x_{2}, x_{3}\right)$ is the electromagnetic wave front, $g_{\alpha \beta}$ is the metric tensor defined in Eq.(120). 
The measurements of spacetime intervals are carried out using light rays and point particles, which are only subject to inertial force and gravitation. Thus, according to Eq.(121) and Eq.(123), the physically observable metric of spacetime, to first order of $f_{0}$, is $g_{\mu \nu}$. Thus, the initial flat background spacetime with metric $\eta_{\mu \nu}$ is no longer physically observable $[34,36,56]$.

If we can further derive the Einstein's equation (1) using the definition (120) of a metric tensor $g_{\mu \nu}$ of a Riemannian spacetime, then, we will provide a geometrical interpretation of Einstein's theory of gravitation in flat spacetime. This is the task of the next section.

\section{DERIVATION OF A GENERALIZED EINSTEIN EQUATION IN INERTIAL COORDINATE SYSTEMS}

Definition 14 The Einstein tensor $G_{\mu \nu}$ is defined by

$$
G_{\mu \nu} \stackrel{\text { def }}{=} R_{\mu \nu}-\frac{1}{2} g_{\mu \nu} R
$$

where $g_{\mu \nu}$ is a metric tensor of a Riemannian spacetime, $R_{\mu \nu}$ is the Ricci tensor, $R \stackrel{\text { def }}{=} g^{\mu \nu} R_{\mu \nu}$.

According to the geometrical interpretation of some theories of gravitation in flat spacetime [34], the physically observable metric $g_{\mu \nu}$ of spacetime in Eq.(120) can be written as

$$
g^{\mu \nu}=\eta^{\mu \nu}-2 f_{0} \psi^{\mu \nu}+O\left(f_{0}\right) .
$$

Following the clue showed in Eq.(125) and noticing the methods of S. N. Gupta [57] and W. Thirring [34], we introduce the following definition of a metric tensor of a Riemannian spacetime.

\section{Definition 15}

$$
\tilde{g}^{\mu \nu} \stackrel{\text { def }}{=} \sqrt{-g_{0}} g^{\mu \nu} \stackrel{\text { def }}{=} \eta^{\mu \nu}-2 f_{0} \phi^{\mu \nu}
$$

where $g_{0}=$ Det $g_{\mu \nu}$.

We have the following expansion of the metric tensor $g^{\mu \nu}[57]$

$$
\begin{aligned}
g^{\mu \nu} & =\eta^{\mu \nu}-2 f_{0} \phi^{\mu \nu}+f_{0} \eta^{\mu \nu} \eta_{\alpha \beta} \phi^{\alpha \beta} \\
& -2 f_{0}^{2} \eta_{\alpha \beta} \phi^{\alpha \beta} \phi^{\mu \nu}+f_{0}^{2} \eta^{\mu \nu} \eta_{\alpha \sigma} \eta_{\beta \lambda} \phi^{\alpha \beta} \phi^{\lambda \sigma} \\
& +\frac{1}{2} f_{0}^{2} \eta^{\mu \nu} \eta_{\alpha \beta} \eta_{\lambda \sigma} \phi^{\alpha \beta} \phi^{\lambda \sigma}+O\left(f_{0}^{3}\right) .
\end{aligned}
$$

Definition 16 If $\phi^{\mu \nu}$ and their first and higher derivatives satisfy the following conditions

$$
\begin{aligned}
& \left|2 f_{0} \phi^{\mu \nu}\right| \ll 1 \\
& \left|\frac{\partial^{n}\left(2 f_{0} \phi^{\mu \nu}\right)}{\partial\left(x^{\alpha}\right)^{n}}\right| \ll 1, n=1,2,3, \cdots
\end{aligned}
$$

then we call this filed $\phi^{\mu \nu}$ weak.

For weak field, $\psi \approx \phi \approx 0$. Thus, $\phi^{\mu \nu}=\psi^{\mu \nu}-\frac{1}{2} \eta^{\mu \nu} \psi \approx \psi^{\mu \nu}$. From Eq.(127), we see that the definition (126) is compatible with Eq.(125).

Theorem 17 Suppose that the conditions in Theorem 8 are valid, then in an arbitrary inertial coordinate system $S_{i}$ we have the following field equation

$$
\begin{aligned}
& G^{\mu \nu}-\frac{1}{2 g_{0}}\left(\sqrt{-g_{0}} g^{\alpha \beta}-\eta^{\alpha \beta}\right) \frac{\partial^{2}\left(\sqrt{-g_{0}} g^{\mu \nu}\right)}{\partial x^{\alpha} \partial x^{\beta}} \\
& -\frac{\sqrt{-g_{0}}}{2 g_{0}}\left(\partial_{\lambda} \partial^{\mu} g^{\nu \lambda}+\partial_{\lambda} \partial^{\nu} g^{\mu \lambda}-\eta^{\mu \nu} \partial_{\sigma} \partial_{\lambda} g^{\sigma \lambda}\right) \\
& -\Pi^{\mu, \alpha \beta} \Pi_{\alpha \beta}^{\nu}+\frac{1}{2} y^{\mu} y^{\nu}-\frac{1}{2} g^{\mu \nu}(L+B) \\
& +B^{\mu \nu}=\frac{f_{0}^{2}}{g_{0}}\left(T^{\mu \nu}-\omega^{\mu \nu}\right)
\end{aligned}
$$


where $T^{\mu \nu}$ is the total energy-momentum tensor of the system of the matter, the $\Omega(1)$ substratum and the $\Omega(0)$ substratum in the inertial coordinate system $S_{i}$,

$$
\begin{aligned}
& \Gamma_{\alpha \beta}^{\nu} \stackrel{\text { def }}{=} \frac{1}{2} g^{\mu \nu}\left(\frac{\partial g^{\mu \alpha}}{\partial x^{\beta}}+\frac{\partial g^{\mu \beta}}{\partial x^{\alpha}}-\frac{\partial g^{\alpha \beta}}{\partial x^{\mu}}\right), \\
& \tilde{g}^{\mu \nu} \stackrel{\text { def }}{=} \sqrt{-g_{0}} g^{\mu \nu}, \\
& \Pi^{\mu, \alpha \beta} \stackrel{\text { def }}{=}-\frac{1}{2 g_{0}}\left(\tilde{g}^{\alpha \lambda} \frac{\partial \tilde{g}^{\mu \beta}}{\partial x^{\lambda}}+\tilde{g}^{\beta \lambda} \frac{\partial \tilde{g}^{\mu \alpha}}{\partial x^{\lambda}}-\tilde{g}^{\mu \lambda} \frac{\partial \tilde{g}^{\alpha \beta}}{\partial x^{\lambda}}\right), \\
& \Pi_{\alpha \beta}^{\nu} \stackrel{\text { def }}{=} g_{\alpha \lambda} g_{\beta \sigma} \Pi^{\nu, \lambda \sigma}, \\
& \Gamma^{\alpha} \stackrel{\text { def }}{=} g^{\sigma \lambda} \Gamma_{\sigma \lambda}^{\alpha} \\
& \Gamma^{\mu \nu} \stackrel{\text { def }}{=} \frac{1}{2}\left(g^{\mu \alpha} \frac{\partial \Gamma^{\nu}}{\partial x^{\alpha}}+g^{\nu \alpha} \frac{\partial \Gamma^{\mu}}{\partial x^{\alpha}}-\frac{\partial g^{\mu \nu}}{\partial x^{\alpha}} \Gamma^{\alpha}\right), \\
& y_{\beta} \stackrel{\text { def }}{=} \frac{\partial\left(\lg \sqrt{-g_{0}}\right)}{\partial x^{\beta}}, y^{\alpha} \stackrel{\text { def }}{=} g^{\alpha \beta} y_{\beta}, \\
& L \stackrel{\text { def }}{=}-\frac{1}{2} \Gamma_{\alpha \beta}^{\nu} \frac{\partial g^{\alpha \beta}}{\partial x^{\nu}}-\Gamma^{\alpha} \frac{\partial\left(\lg \sqrt{-g_{0}}\right)}{\partial x^{\alpha}}, \\
& B^{\mu \nu} \stackrel{\text { def }}{=} \Gamma^{\mu \nu}+\frac{1}{2}\left(y^{\mu} \Gamma^{\nu}+y^{\nu} \Gamma^{\mu}\right), B \stackrel{\text { def }}{=} g_{\mu \nu} B^{\mu \nu} .
\end{aligned}
$$

Proof of Theorem 17. According to a theorem of V. Fock ([50], p429), the Einstein tensor $G^{\mu \nu}$ can be written as

$$
\begin{aligned}
G^{\mu \nu}= & \frac{1}{2 g_{0}} \tilde{g}^{\alpha \beta} \frac{\partial^{2} \tilde{g}^{\mu \nu}}{\partial x_{\alpha} \partial x_{\beta}}+\Pi^{\mu, \alpha \beta} \Pi_{\alpha \beta}^{\nu}-\frac{1}{2} y^{\mu} y^{\nu} \\
& +\frac{1}{2} g^{\mu \nu}(L+B)-B^{\mu \nu} .
\end{aligned}
$$

Applying Eq.(126), Eq.(140) can be written as

$$
\begin{aligned}
G^{\mu \nu}= & \frac{1}{2 g_{0}} \sqrt{-g_{0}} g^{\alpha \beta} \frac{\partial^{2}\left(\eta^{\mu \nu}-2 f_{0} \phi^{\mu \nu}\right)}{\partial x^{\alpha} \partial x^{\beta}}+\Pi^{\mu, \alpha \beta} \Pi_{\alpha \beta}^{\nu} \\
& -\frac{1}{2} y^{\mu} y^{\nu}+\frac{1}{2} g^{\mu \nu}(L+B)-B^{\mu \nu} \\
= & \frac{1}{2 g_{0}}\left(\sqrt{-g_{0}} g^{\alpha \beta}-\eta^{\alpha \beta}+\eta^{\alpha \beta}\right) \frac{\partial^{2}\left(-2 f_{0} \phi^{\mu \nu}\right)}{\partial x^{\alpha} \partial x^{\beta}} \\
& +\Pi^{\mu, \alpha \beta} \Pi_{\alpha \beta}^{\nu}-\frac{1}{2} y^{\mu} y^{\nu}+\frac{1}{2} g^{\mu \nu}(L+B)-B^{\mu \nu} \\
= & \frac{1}{2 g_{0}}\left(\sqrt{-g_{0}} g^{\alpha \beta}-\eta^{\alpha \beta}\right) \frac{\partial^{2}\left(-2 f_{0} \phi^{\mu \nu}\right)}{\partial x^{\alpha} \partial x^{\beta}} \\
& -\frac{f_{0}}{g_{0}} \eta^{\alpha \beta} \frac{\partial^{2} \phi^{\mu \nu}}{\partial x^{\alpha} \partial x^{\beta}}+\Pi^{\mu, \alpha \beta} \Pi_{\alpha \beta}^{\nu} \\
& -\frac{1}{2} y^{\mu} y^{\nu}+\frac{1}{2} g^{\mu \nu}(L+B)-B^{\mu \nu} .
\end{aligned}
$$


Noticing Eq.(126), the field equation Eq.(115) can be written as

$$
\begin{aligned}
\eta^{\alpha \beta} \frac{\partial^{2} \phi^{\mu \nu}}{\partial x^{\alpha} \partial x^{\beta}} & =-\frac{\sqrt{-g_{0}}}{2 f_{0}}\left(\partial_{\lambda} \partial^{\mu} g^{\nu \lambda}+\partial_{\lambda} \partial^{\nu} g^{\mu \lambda}\right. \\
& \left.-\eta^{\mu \nu} \partial_{\sigma} \partial_{\lambda} g^{\sigma \lambda}\right)-f_{0}\left(T^{\mu \nu}-\omega^{\mu \nu}\right) .
\end{aligned}
$$

Using Eq.(126) and Eq.(142), Eq.(141) can be written as

$$
\begin{aligned}
G^{\mu \nu}= & \frac{1}{2 g_{0}}\left(\sqrt{-g_{0}} g^{\alpha \beta}-\eta^{\alpha \beta}\right) \frac{\partial^{2}\left(\sqrt{-g_{0}} g^{\mu \nu}\right)}{\partial x^{\alpha} \partial x^{\beta}} \\
& +\frac{\sqrt{-g_{0}}}{2 g_{0}}\left(\partial_{\lambda} \partial^{\mu} g^{\nu \lambda}+\partial_{\lambda} \partial^{\nu} g^{\mu \lambda}-\eta^{\mu \nu} \partial_{\sigma} \partial_{\lambda} g^{\sigma \lambda}\right) \\
& +\frac{f_{0}^{2}}{g_{0}}\left(T^{\mu \nu}-\omega^{\mu \nu}\right)+\Pi^{\mu, \alpha \beta} \Pi_{\alpha \beta}^{\nu}-\frac{1}{2} y^{\mu} y^{\nu} \\
& +\frac{1}{2} g^{\mu \nu}(L+B)-B^{\mu \nu} .
\end{aligned}
$$

Eq.(143) can be written as Eq.(130). Proof ends.

Eq.(130) has the same form in all inertial coordinate systems. Eq.(130) is one of the main results in this manuscript. We need to further study the relationship between Eq.(130) and the Einstein field equation Eq.(1).

If we impose the Hilbert gauge condition Eq.(98) on the fields, then we have the following result.

Theorem 18 Suppose that the conditions in Proposition 9 are valid, then in an arbitrary inertial coordinate system $S_{i}$ we have the following field equation

$$
\begin{aligned}
& G^{\mu \nu}-\frac{1}{2 g_{0}}\left(\sqrt{-g_{0}} g^{\alpha \beta}-\eta^{\alpha \beta}\right) \frac{\partial^{2}\left(\sqrt{-g_{0}} g^{\mu \nu}\right)}{\partial x^{\alpha} \partial x^{\beta}} \\
& -\Pi^{\mu, \alpha \beta} \Pi_{\alpha \beta}^{\nu}+\frac{1}{2} y^{\mu} y^{\nu}-\frac{1}{2} g^{\mu \nu}(L+B) \\
& +B^{\mu \nu}=\frac{f_{0}^{2}}{g_{0}}\left(T^{\mu \nu}-\omega^{\mu \nu}\right) .
\end{aligned}
$$

Proof of Theorem 18. Using Eq.(126) and Eq.(107), Eq.(141) can be written as

$$
\begin{aligned}
G^{\mu \nu}= & \frac{1}{2 g_{0}}\left(\sqrt{-g_{0}} g^{\alpha \beta}-\eta^{\alpha \beta}\right) \frac{\partial^{2}\left(\sqrt{-g_{0}} g^{\mu \nu}\right)}{\partial x^{\alpha} \partial x^{\beta}} \\
& -\frac{f_{0}^{2}}{g_{0}} T^{\mu \nu}+\Pi^{\mu, \alpha \beta} \Pi_{\alpha \beta}^{\nu}-\frac{1}{2} y^{\mu} y^{\nu} \\
& +\frac{1}{2} g^{\mu \nu}(L+B)-B^{\mu \nu} .
\end{aligned}
$$

Eq.(145) can be written as Eq.(144). Proof ends.

Definition 19 If each of the coordinates $x^{\alpha}$ satisfies the following generalized wave equation

$$
\frac{1}{\sqrt{-g_{0}}} \frac{\partial}{\partial x^{\mu}}\left(\sqrt{-g_{0}} g^{\mu \nu} \frac{\partial x^{\alpha}}{\partial x^{\nu}}\right)=0
$$

then, we call such a coordinates system harmonic.

In a harmonic coordinates system, we have ([50], p254)

$$
\Gamma^{\nu}=\Gamma^{\mu \nu}=B^{\mu \nu}=B=0 .
$$

Putting Eq.(147) into Eq.(144), we have the following corollary.

Corollary 20 Suppose that the conditions in Proposition 9 are valid and the coordinates system is harmonic, then the field equation Eq.(144) can be written as

$$
\begin{aligned}
& G^{\mu \nu}-\frac{1}{2 g_{0}}\left(\sqrt{-g_{0}} g^{\alpha \beta}-\eta^{\alpha \beta}\right) \frac{\partial^{2}\left(\sqrt{-g_{0}} g^{\mu \nu}\right)}{\partial x^{\alpha} \partial x^{\beta}} \\
& -\Pi^{\mu, \alpha \beta} \Pi_{\alpha \beta}^{\nu}+\frac{1}{2} y^{\mu} y^{\nu}-\frac{1}{2} g^{\mu \nu} L \\
& =\frac{f_{0}^{2}}{g_{0}}\left(T^{\mu \nu}-\omega^{\mu \nu}\right) .
\end{aligned}
$$


We can verify that each of the Galilean coordinates is harmonic. Any constant and any linear function of harmonic coordinates satisfy Eq.(146). Thus, from Eq.(106), an inertial coordinate system is harmonic and Eq.(148) is valid.

Assumption 21 For the weak fields, the following relationship is valid

$$
T^{\mu \nu}-\omega^{\mu \nu} \approx T_{m}^{\mu \nu}
$$

Corollary 22 Suppose that (1) the conditions in Proposition 9 are valid; (2) the filed is weak; (3) Assumption 21 is valid. Then in an arbitrary inertial coordinate system the field equation Eq.(148) reduces to

$$
R_{\mu \nu}-\frac{1}{2} g_{\mu \nu} R=\frac{f_{0}^{2}}{g_{0}} T_{\mu \nu}^{m}
$$

Proof of Corollary 22. According to Definition 16, $f_{0} \phi^{\mu \nu}$ and their first and higher derivatives are small quantities of order $\varepsilon$, where $|\varepsilon| \ll 1$ is a small quantity. Thus, from Eq.(127), we have the following estimation of the order of magnitude of the following quantities

$$
\sqrt{-g_{0}} g^{\mu \nu}-\eta^{\mu \nu} \sim \varepsilon, \frac{\partial g_{\mu \nu}}{\partial x^{\alpha}} \sim \frac{\partial g^{\mu \nu}}{\partial x^{\alpha}} \sim \varepsilon .
$$

Using Eq.(126), we have the following estimation of the order of magnitude of the quantity

$$
\frac{\partial^{2}\left(\sqrt{-g_{0}} g^{\alpha \beta}\right)}{\partial x^{\alpha} \partial x^{\beta}}=\frac{\partial^{2}\left(-2 f_{0} \phi^{\mu \nu}\right)}{\partial x^{\alpha} \partial x^{\beta}} \sim \varepsilon
$$

Thus, from Eq.(151) and Eq.(152), we have the following estimation of the order of magnitude of the quantity

$$
\left(\sqrt{-g_{0}} g^{\alpha \beta}-\eta^{\alpha \beta}\right) \frac{\partial^{2}\left(\sqrt{-g_{0}} g^{\alpha \beta}\right)}{\partial x^{\alpha} \partial x^{\beta}} \sim \varepsilon^{2} .
$$

Using Eq.(133) and Eq.(134), we have the following estimation of the order of magnitude of the quantity

$$
\Pi^{\mu, \alpha \beta} \sim \Pi_{\alpha \beta}^{\nu} \sim \varepsilon
$$

From Eq.(137), we have the following relationship ([50], p143)

$$
y_{\beta}=\Gamma_{\beta \nu}^{\nu} .
$$

We also have ([50], p143)

$$
\Gamma_{\beta \nu}^{\nu}=\frac{1}{2} g^{\mu \nu} \frac{\partial g_{\mu \nu}}{\partial x^{\beta}}
$$

From Eq.(155), Eq.(156) and Eq.(151), we have the following estimation of the order of magnitude

$$
y_{\beta} \sim \varepsilon .
$$

Using Eq.(137) and Eq.(157), we have the following estimation of the order of magnitude

$$
y^{\alpha} \sim \varepsilon
$$

From Eq.(138) and Eq.(147), we have

$$
L=-\frac{1}{2} \Gamma_{\alpha \beta}^{\nu} \frac{\partial g^{\alpha \beta}}{\partial x^{\alpha}} .
$$

Using Eq.(159), Eq.(156) and Eq.(151), we have the following estimation of the order of magnitude

$$
L \sim \varepsilon^{2} .
$$

From Eq.(153), Eq.(154), Eq.(158) and Eq.(160), we see that the second to the fifth term on the right side of Eq.(148) are all small quantities of order $\varepsilon^{2}$. Ignoring all these small quantities of order $\varepsilon^{2}$ in Eq.(148) and using Eq.(149), we obtain

$$
G^{\mu \nu} \approx \frac{f_{0}^{2}}{g_{0}} T_{m}^{\mu \nu}
$$


Applying the rules of lowering or raising the indexes of tensors, i.e., $G^{\mu \nu}=g^{\mu \lambda} g^{\nu \sigma} G_{\lambda \sigma}, T_{m}^{\mu \nu}=g^{\mu \lambda} g^{\nu \sigma} T_{\lambda \sigma}^{m}$, Eq.(161) can be written as

$$
G_{\lambda \sigma} \approx \frac{f_{0}^{2}}{g_{0}} T_{\lambda \sigma}^{m}
$$

Putting Eq.(124) into Eq.(162), we obtain Eq.(150). Proof ends.

Corollary 23 Suppose that the following conditions are valid: (1) the conditions of Proposition 9 are valid; (2) the filed is weak; (3) $g^{\mu \nu} \approx \eta^{\mu \nu}$; (4) Assumption 21 is valid. Then in an arbitrary inertial coordinate system the field equation Eq.(150) reduces to

$$
R_{\mu \nu}-\frac{1}{2} g_{\mu \nu} R=-f_{0}^{2} T_{\mu \nu}^{m}
$$

Proof of Corollary 23. Since $g^{\mu \nu} \approx \eta^{\mu \nu}, g_{\mu \nu} \approx \eta_{\mu \nu}$, we have $g_{0}=\operatorname{det} g_{\mu \nu} \approx \operatorname{det} \eta_{\mu \nu}=-1$. Thus, Eq.(150) can be written as Eq.(163). Proof ends.

If we introduce the following notation

$$
\kappa=f_{0}^{2}=\frac{8 \pi \gamma_{N}}{c^{4}}
$$

then, Eq.(163) coincides with Einstein's equation Eq.(1). Thus, we see that the field equation Eq.(130) is a generalization of the Einstein's equation Eq.(1) in inertial coordinate systems.

\section{DERIVATION OF A GENERALIZED EINSTEIN EQUATION IN SOME NON-INERTIAL COORDINATE SYSTEMS}

Now we consider an arbitrary non-inertial coordinate system $S^{n}$. We introduce an arbitrary curvilinear coordinates $\left(z_{0}, z_{1}, z_{2}, z_{3}\right)$ in the non-inertial coordinate system $S^{n}$. We do not know whether Theorem 18 is valid or not in the non-inertial coordinate system $S^{n}$.

Assumption 24 Suppose that in a non-inertial coordinate system $S^{n}$ there exists a symmetric tensor $\phi_{\mu \nu}$ which satisfies the following wave equation

$$
\gamma^{\alpha \beta} \frac{\partial^{2} \phi^{\mu \nu}}{\partial z_{\alpha} \partial z_{\beta}}=-f_{0}\left(T^{\mu \nu}-\omega^{\mu \nu}\right)
$$

where $\gamma_{\mu \nu}$ is the metric tensor of the non-inertial coordinate system $S^{n}$.

We introduce the following definition of a metric tensor of a Riemannian spacetime.

\section{Definition 25}

$$
\tilde{g}^{\mu \nu} \stackrel{\text { def }}{=} \sqrt{-g_{0}} g^{\mu \nu} \stackrel{\text { def }}{=} \gamma^{\mu \nu}-2 f_{0} \phi^{\mu \nu}
$$

where $g_{0}=$ Det $g_{\mu \nu}, f_{0}$ is a parameter to be determined.

Theorem 26 Suppose that Assumption 24 is valid. Then, in the non-inertial coordinate system $S^{n}$, we have the following field equation

$$
\begin{aligned}
& G^{\mu \nu}-\frac{1}{2 g_{0}}\left(\sqrt{-g_{0}} g^{\alpha \beta}-\gamma^{\alpha \beta}\right) \frac{\partial^{2}\left(\sqrt{-g_{0}} g^{\mu \nu}\right)}{\partial z_{\alpha} \partial z_{\beta}} \\
& +\frac{1}{2 g_{0}} \gamma^{\alpha \beta} \frac{\partial^{2} \gamma^{\mu \nu}}{\partial z_{\alpha} \partial z_{\beta}}-\Pi^{\mu, \alpha \beta} \Pi_{\alpha \beta}^{\nu}+\frac{1}{2} y^{\mu} y^{\nu} \\
& -\frac{1}{2} g^{\mu \nu}(L+B)+B^{\mu \nu}=\frac{f_{0}^{2}}{g_{0}}\left(T^{\mu \nu}-\omega^{\mu \nu}\right),
\end{aligned}
$$

where $T_{\mu \nu}=T_{\mu \nu}^{m}+T_{\mu \nu}^{\Omega(1)}+T_{\mu \nu}^{\Omega(0)}$ is total energy-momentum tensor of the matter, the $\Omega(1)$ substratum and the $\Omega(0)$ substratum in the non-inertial coordinate system $S^{n}$. 
Proof of Theorem 26. Using Eq.(166), Eq.(140) can be written as

$$
\begin{aligned}
G^{\mu \nu}= & \frac{1}{2 g_{0}} \sqrt{-g_{0}} g^{\alpha \beta} \frac{\partial^{2}\left(\gamma^{\mu \nu}-2 f_{0} \phi^{\mu \nu}\right)}{\partial z_{\alpha} \partial z_{\beta}}+\Pi^{\mu, \alpha \beta} \Pi_{\alpha \beta}^{\nu} \\
& -\frac{1}{2} y^{\mu} y^{\nu}+\frac{1}{2} g^{\mu \nu}(L+B)-B^{\mu \nu} \\
= & \frac{1}{2 g_{0}}\left(\sqrt{-g_{0}} g^{\alpha \beta}-\gamma^{\alpha \beta}+\gamma^{\alpha \beta}\right) \frac{\partial^{2}\left(-2 f_{0} \phi^{\mu \nu}\right)}{\partial z_{\alpha} \partial z_{\beta}} \\
& +\Pi^{\mu, \alpha \beta} \Pi_{\alpha \beta}^{\nu}-\frac{1}{2} y^{\mu} y^{\nu}+\frac{1}{2} g^{\mu \nu}(L+B)-B^{\mu \nu} \\
= & \frac{1}{2 g_{0}}\left(\sqrt{-g_{0}} g^{\alpha \beta}-\gamma^{\alpha \beta}\right) \frac{\partial^{2}\left(-2 f_{0} \phi^{\mu \nu}\right)}{\partial z_{\alpha} \partial z_{\beta}} \\
& -\frac{f_{0}}{g_{0}} \gamma^{\alpha \beta} \frac{\partial^{2} \phi^{\mu \nu}}{\partial z_{\alpha} \partial z_{\beta}}+\Pi^{\mu, \alpha \beta} \Pi_{\alpha \beta}^{\nu} \\
& -\frac{1}{2} y^{\mu} y^{\nu}+\frac{1}{2} g^{\mu \nu}(L+B)-B^{\mu \nu} .
\end{aligned}
$$

Using Eq.(166) and Eq.(165), Eq.(168) can be written as Eq.(167). Proof ends.

We need to study the relationship between Eq.(167) and the Einstein field equation (1). Using Eq.(147) and Eq.(167), we have the following corollary.

Corollary 27 Suppose that the conditions in Theorem 26 are valid and the coordinate system is harmonic, then in the non-inertial coordinate system $S^{n}$ the field equation Eq.(167) can be written as

$$
\begin{aligned}
& G^{\mu \nu}-\frac{1}{2 g_{0}}\left(\sqrt{-g_{0}} g^{\alpha \beta}-\gamma^{\alpha \beta}\right) \frac{\partial^{2}\left(\sqrt{-g_{0}} g^{\mu \nu}\right)}{\partial z_{\alpha} \partial z_{\beta}} \\
& +\frac{1}{2 g_{0}} \gamma^{\alpha \beta} \frac{\partial^{2} \gamma^{\mu \nu}}{\partial z_{\alpha} \partial z_{\beta}}-\Pi^{\mu, \alpha \beta} \Pi_{\alpha \beta}^{\nu} \\
& +\frac{1}{2} y^{\mu} y^{\nu}-\frac{1}{2} g^{\mu \nu} L=\frac{f_{0}^{2}}{g_{0}}\left(T^{\mu \nu}-\omega^{\mu \nu}\right)
\end{aligned}
$$

Definition 28 If we have the following conditions

$$
\begin{aligned}
& \left|\gamma^{\mu \nu}-\eta^{\mu \nu}\right| \ll 1 \\
& \left|\frac{1}{2} \gamma^{\alpha \beta} \frac{\partial^{2} \gamma^{\mu \nu}}{\partial z_{\alpha} \partial z_{\beta}}\right| \ll\left|f_{0}^{2}\left(T^{\mu \nu}-\omega^{\mu \nu}\right)\right|,
\end{aligned}
$$

then we call this non-inertial coordinate system $S^{n}$ quasi-inertial.

Assumption 29 For weak fields in the non-inertial coordinate system $S^{n}$, the following relationship is valid

$$
T^{\mu \nu}-\omega^{\mu \nu} \approx T_{m}^{\mu \nu}
$$

Using Eq.(172), Eqs.(170-171) and Eq.(169), we have the following corollary.

Corollary 30 Suppose that (1) the conditions in Theorem 26 are valid; (2) the coordinate system is harmonic; (3) the non-inertial coordinate system $S^{n}$ is quasi-inertial, then the field equation Eq.(169) can be written as

$$
\begin{aligned}
& G^{\mu \nu}-\frac{1}{2 g_{0}}\left(\sqrt{-g_{0}} g^{\alpha \beta}-\eta^{\alpha \beta}\right) \frac{\partial^{2}\left(\sqrt{-g_{0}} g^{\alpha \beta}\right)}{\partial z_{\alpha} \partial z_{\beta}} \\
& -\Pi^{\mu, \alpha \beta} \Pi_{\alpha \beta}^{\nu}+\frac{1}{2} y^{\mu} y^{\nu}-\frac{1}{2} g^{\mu \nu} L=\frac{f_{0}^{2}}{g_{0}} T^{\mu \nu} .
\end{aligned}
$$

Eq.(173) is only valid approximately in a quasi-inertial and harmonic coordinate system $S^{n}$. Similar to Corollary 22 , we have the following corollary.

Corollary 31 Suppose that (1) the conditions in Theorem 26 are valid; (2) the coordinate system is harmonic; (3) the non-inertial coordinate system $S^{n}$ is quasi-inertial; (4) the filed is weak. Then, the field equation Eq.(173) reduces to

$$
R_{\mu \nu}-\frac{1}{2} g_{\mu \nu} R=\frac{f_{0}^{2}}{g_{0}} T_{\mu \nu}
$$


Similar to Corollary 23, we have the following corollary.

Corollary 32 Suppose that (1) the conditions in Theorem 26 are valid; (2) the coordinate system is harmonic; (3) the non-inertial coordinate system $S^{n}$ is weak; (4) the filed is weak; (5) $g^{\mu \nu} \approx \eta^{\mu \nu} ;(6) T_{\mu \nu}^{\Omega(1)} \ll T_{\mu \nu}^{m}$; (7) $T_{\mu \nu}^{\Omega(0)} \ll T_{\mu \nu}^{m}$. Then, in this weak non-inertial coordinate system $S^{n}$ the field equation Eq.(174) reduces to

$$
R_{\mu \nu}-\frac{1}{2} g_{\mu \nu} R=-f_{0}^{2} T_{\mu \nu}^{m}
$$

Comparing Eq.(175) and Eq.(1), we see that the field equation Eq.(167) is a generalization of the Einstein's equation Eq.(1) in some special non-inertial coordinate systems.

\section{DISCUSSION}

Although the field equation Eq.(130) and Eq.(167) are generalizations of the Einstein's equation (1), there exists at least the following 8 differences between this theory and Einstein's theory of general relativity.

(1). In Einstein's theory, Einstein's equation (1) is an assumption [1-3]. Although Einstein introduced his new concept of gravitational aether ([24], p63-113), Einstein did not derive his equation (1) theoretically based on his new concept of the gravitational aether. In our theory, the two generalized Einstein's equation Eq.(130) and Eq.(167) are derived by methods of special relativistic continuum mechanics based on some assumptions.

(2). Although the theory of general relativity is a field theory of gravity, the definitions of gravitational fields are not based on continuum mechanics [1-3, 58-61]. Because of the absence of a continuum, the theory of general relativity may be regarded as a phenomenological theory of gravity [1-3]. In our theory, gravity is transmitted by the $\Omega(0)$ substratum. The tensorial potential $\psi_{\mu \nu}$ of gravitational fields are defined based on special relativistic continuum mechanics.

(3). In Einstein's theory, the concept of Riemannian spacetime is introduced together with the field equation (1) [1-3]. The theory of general relativity can not provide a physical definition of the metric tensor of the Riemannian spacetime. In our theory, the background spacetime is the Minkowshi spacetime. However, the initial flat background spacetime with metric $\eta_{\mu \nu}$ is no longer physically observable. According to the equation of motion (121) of a point particle in gravitational field, to first order of $f_{0}$, the physically observable spacetime is a Riemannian spacetime with the metric tensor $g_{\mu \nu}$. The metric tensor $g_{\mu \nu}$ is defined based on the tensorial potential $\psi_{\mu \nu}$ of gravitational fields.

(4). The masses of particles are constants in Einstein's theory of general relativity [1-3]. In our theory, the masses of particles are functions of time $t[38]$.

(5). The gravitational constant $\gamma_{N}$ is a constant in Einstein's theory of general relativity [1-3]. The theory of general relativity can not provide a derivation of $\gamma_{N}$. In our theory, the parameter $\gamma_{N}$ is derived theoretically. From Eq.(6), we see that $\gamma_{N}$ depends on time $t$.

(6). In our theory, parameter $\gamma_{N}$ in Eq.(11) depends on the density $\rho_{0}$ of the $\Omega(0)$ substratum. If $\rho_{0}$ varies from place to place, i.e., $\rho=\rho(\mathbf{r})$, then the space dependence of the gravitational constant $\gamma_{N}$ can be seen from Eq. $(6)$.

(7). In Einstein's theory, equation (1) is supposed to be valid in all coordinate systems [1-3]. In our theory, the generalized Einstein's equation Eq.(130) is valid only in inertial coordinate systems. The second generalized Einstein's equation Eq.(167) is valid only in some special non-inertial coordinate systems.

(8). In Einstein's theory, equation (1) is rigorous [1-3]. However, in our theory, Eq.(175) is valid approximately under some assumptions.

\section{CONCLUSION}

Einstein's field equation is a fundamental assumption in general relativity. The theory of general relativity is facing some puzzles and difficulties. We extend our previous theory of gravitation to the case of special relativistic fluid mechanics. In inertial coordinate systems, we construct a tensorial potential which satisfies the wave equation. Inspired by the equation of motion of a test particle, a definition of a metric tensor of a Riemannian spacetime is introduced. A generalized Einstein's equation is derived in inertial coordinate systems based on some assumptions. This equation reduces to Einstein's equation in case of weak field in harmonic coordinate systems. In some special non-inertial coordinate systems, a second generalized Einstein's equation is derived based on some assumptions. If the field is weak and the coordinate system is quasi-inertial and harmonic, the second generalized Einstein's equation reduces to Einstein's equation. Thus, this theory also explains all the experiments that support the theory of general relativity. However, because of the absence of a continuum, the theory of general relativity may be regarded as a phenomenological theory of gravity. Such kinds of phenomenological theories can not provide a physical definition 
of the tensorial potential of gravitational fields. In our theory, gravity is transmitted by the $\Omega(0)$ substratum. The tensorial potential of gravitational fields are defined based on special relativistic continuum mechanics. In Einstein's theory, the concept of Riemannian spacetime is introduced together with the field equation. In our theory, the background spacetime is the Minkowshi spacetime. However, the initial flat background spacetime is no longer physically observable. According to the equation of motion of a point particle in gravitational field, to first order, the physically observable spacetime is a Riemannian spacetime. The metric tensor of this Riemannian spacetime is defined based on the tensorial potential of gravitational fields.

\section{ACKNOWLEDGMENTS}

I am grateful to Prof. Ru-Zeng Zhu for his criticisms on my previous theory of gravitation [38].

[1] C. Møller, The theory of relativity (Clarendon Press, Oxford, 1955).

[2] S. Weinberg, Gravitation and cosmology (John Wiley \& sons, New York, 1972).

[3] C. Misner, K. Thorne, and J. Wheeler, Gravitation (Freeman, New York, 1973).

[4] P. Dirac, General theory of relativity (Princeton University Press, Princeton, 1996).

[5] M. Kramer and et al., Science 314, 97 (2006).

[6] C. W. Will, Living Rev. Relativity 17, 4 (2014).

[7] R. D. Blandford, Science 347, 1103 (2015).

[8] B. P. Abbott and et al., Phys. Rev. Lett. 116, 221101 (2016).

[9] A. Hees and et al., Phys. Rev. Lett. 118, 211101 (2017).

[10] R. P. Feynman, Feynman lectures on gravitation, foreword by John Preskill and Kip S. Thorne (Addison-Wesley Publishing Company, New York, 1995).

[11] J. Maddox, What remains to be discovered (The Free Press, New York, 1998).

[12] D. Marsh, Phys. Rev. Lett. 118, 011302 (2017).

[13] A. Cho, Science 355, 560 (2017).

[14] A. Einstein, Ann. Math 40, 922 (1939).

[15] P. Dirac, Directions in Physics (John Wiley \& sons, New York, 1978).

[16] P. Hamilton, M. Jaffe, P. Haslinger, Q. Simmons, H. Müller, and J. Khoury, Science 349, 849 (2015).

[17] D. Spergel, Science 347, 1100 (2015).

[18] J. Kim, P. Sikivie, and S. Weinberg, Nature 541, 464 (2017).

[19] A. Bosma, Nature 543, 179 (2017).

[20] E. Gibney, Nature 551, 153 (2017).

[21] E. Whittaker, A History of the Theories of Aether and Electricity, Revised and enlarged edtion, vol. 1 (Thomas Nelson and Sons Ltd., London, 1951).

[22] T. Hirosige, History of physics, in Chinese, translated from the Japanese edtion published in 1968 (1968).

[23] I. Lakatos, The methodology of scientific research programmes, Philosophical Papers, Volume I (Cambridge University Press, New York, 1978).

[24] L. Kostro, Einstein and the Ether (Apeiron, Montreal, 2000).

[25] P. A. M. Dirac, Direction in Physics (Wiley, New York, 1978).

[26] D. R. Long, Nature 260, 417 (1976).

[27] D. Mugnai, A. Ranfagni, and R. Ruggeri, Phys. Rev. Lett. 84, 4830 (2000).

[28] D. Giovannini, J. Romero, V. Potocek, G. Ferenczi, F. Speirits, S. Barnett, D. Faccio, and M. Padgett, Science 347, 857 (2015).

[29] J. R. Sambles, Science 347, 828 (2015).

[30] N. Cornish, D. Blas, and G. Nardini, Phys. Rev. Lett. 119, 161102 (2017).

[31] A. Einstein, Letters to Solovine 1906-1955 (Citadel, 2000).

[32] S. N. Gupta, Phys. Rev. 96, 1683 (1954).

[33] R. H. Kraichnan, Physical Review 98, 1118 (1955).

[34] W. Thirring, Annals of Physics 16, 96 (1961).

[35] S. Weinberg, Physical Review 138, B988 (1965).

[36] S. Deser, General Relativity and Gravitation 1, 9 (1970).

[37] S. V. Babak and L. P. Grishchuk, Physical Review D 61, 024038 (1999).

[38] X.-S. Wang, Progress in Physics 4, 25 (2008).

[39] B. Riemann, Collected Papers, Translated from the 1892 edition by Roger Baker, Charles Christenson and Henry Orde (Kendrick Press, Heber, 2004).

[40] H. Poincare, The science and hypothesis, translated from the French edtion, in Chinese (Shangwu Press, Beijing, 1997).

[41] A. Einstein and L. Infeld, The evolution of physics (Cambridge University Press, London, 1938). 
[42] W.-Y. Wu, Fluid Mechanics, vol. 1, in Chinese (Beijing University Press, Beijing, 1982).

[43] T. E. Faber, Fluid Dynamics for Physicists (Cambridge University Press, Cambridge, 1995).

[44] I. G. Currie, Fundamental Mechanics of Fluids, 3rd ed. (Cambridge University Press, 2003).

[45] I. Newton, The Principia Mathematical Principles of Natural Philosophy, A New Translation by I. Bernard Cohen and Anne Whitman assisted by Julia Budenz (University Of California Press, Berkeley, 1999).

[46] E. Whittaker, A History of the Theories of Aether and Electricity (Thomas Nelson and Sons Ltd., London, 1953).

[47] X.-S. Wang, Progress in Physics 2, 111 (2008).

[48] X.-S. Wang, Physics Essays 27, 398 (2014).

[49] W. Rindler, Introduction to special relativity (Clarendon Press, Oxford, 1982).

[50] V. Fock, The Theory of Space Time and Gravitation, 2nd Revised Edition, Translated from the Russian by N. Kemmer (Pergamon Press, London, 1964).

[51] Z.-D. Chen, Rational mechanics, in Chinese (2000).

[52] Y.-H. Pao and C.-C. Mow, Diffraction of Elastic Waves and Dynamic Stress Concentrations (Crane, Russak Company Inc. in US, 1973).

[53] A. C. Eringen, Elastodynamics (Academic Press, New York, 1975).

[54] H. C. Ohanian and R. Ruffini, Gravitation and Spacetime, 3rd edition (Cambridge University Press, New York, 2013).

[55] L. Liu, The theory of general relativity, in Chinese (Advanced Education Press, Beijing, 2004).

[56] P. Mittelstaedt and J. B. Barbour, Zeitschrift für Physik 203, 82 (1967).

[57] S. N. Gupta, Proc. Phys. Soc. A65, 608 (1952).

[58] C. Truesdell, The Elements of Continuum Mechanics (Springer-Verlag, New York, 1966).

[59] Y. C. Fung, A First Course in Continuum Mechanics (Prentice-Hall, London, 1977).

[60] A. C. Eringen, The Elements of Continuum Mechanics (Robert E. Krieger Pub. Co., Huntington, 1980).

[61] L. D. Landau and Lifshitz, Fluid Mechanics, translated from the Russian by J.B. Sykes and W.H. Reid. (Pergamon, New York, 1987). 